\title{
SULT4A1 Modulates Synaptic Development and Function by Promoting the Formation of PSD-95/NMDAR Complex
}

\author{
Lorenza Culotta, ${ }^{1}{ }^{\circledR}$ Paolo Scalmani, ${ }^{2}$ Ersilia Vinci, ${ }^{1}$ Benedetta Terragni, ${ }^{2}$ Alessandro Sessa, ${ }^{3}$ Vania Broccoli, ${ }^{1}$ \\ (D) Massimo Mantegazza, ${ }^{4}$ Tobias Boeckers, ${ }^{5,6}$ and ${ }^{(D)}$ Chiara Verpelli ${ }^{1}$ \\ ${ }^{1}$ CNR Neuroscience Institute, 20129 Milan, Italy, ${ }^{2}$ L'Unità Operativa Complessa di Epilettologia Clinica e Sperimentale, Foundation Istituto di \\ Ricerca e Cura a Carattere Scientifico (IRCCS), Neurological Institute Carlo Besta, 20133 Milan, Italy, ${ }^{3}$ Stem Cell and Neurogenesis Unit, Division of \\ Neuroscience, San Raffaele Scientific Institute, 20132 Milan, Italy, ${ }^{4}$ CNRS UMR 7275, Institut National de la Santé et de la Recherche Médicale, \\ LabEx ICST, Institute of Molecular and Cellular Pharmacology (IPMC), Université Côte d'Azur (UCA), 06560 Valbonne-Sophia Antipolis, France, \\ ${ }^{5}$ Institute for Anatomy and Cell Biology, Ulm University, 89081 Ulm, Germany, and ${ }^{6}$ DZNE, Ulm Site, 89081 Ulm, Germany
}

Sulfotransferase 4A1 (SULT4A1) is a cytosolic sulfotransferase that is highly conserved across species and extensively expressed in the brain. However, the biological function of SULT4A1 is unclear. SULT4A1 has been implicated in several neuropsychiatric disorders, such as Phelan-McDermid syndrome and schizophrenia. Here, we investigate the role of SULT4A1 within neuron development and function. Our data demonstrate that SULT4A1 modulates neuronal branching complexity and dendritic spines formation. Moreover, we show that SULT4A1, by negatively regulating the catalytic activity of Pin1 toward PSD-95, facilitates NMDAR synaptic expression and function. Finally, we demonstrate that the pharmacological inhibition of Pin1 reverses the pathologic phenotypes of neurons knocked down by SULT4A1 by specifically restoring dendritic spine density and rescuing NMDAR-mediated synaptic transmission. Together, these findings identify SULT4A1 as a novel player in neuron development and function by modulating dendritic morphology and synaptic activity.

Key words: dendrites; Pin1; postsynaptic density; synapse

Significance Statement

Sulfotransferase 4A1 (SULT4A1) is a brain-specific sulfotransferase highly expressed in neurons. Different evidence has suggested that SULT4A1 has an important role in neuronal function and that SULT4A1 altered expression might represent a contributing factor in multiple neurodevelopmental disorders. However, the function of SULT4A1 in the mammalian brain is still unclear. Here, we demonstrate that SULT4A1 is highly expressed at postsynaptic sites where it sequesters Pin1, preventing its negative action on synaptic transmission. This study reveals a novel role of SULT4A1 in the modulation of NMDA receptor activity and strongly contributes to explaining the neuronal dysfunction observed in patients carrying deletions of SULTA41 gene.

\section{Introduction}

Cytosolic sulfotransferase 4A1 (SULT4A1) is member of the superfamily of cytosolic sulfotransferases, a class of enzymes that catalyze sulfonation reactions by transferring the sulfonate group from 3 '-phosphoadenosine $5^{\prime}$-phosphosulfate (PAPS) to different endogenous and exogenous substrates (Falany et al., 2000; Negishi et al., 2001). SULT4A1 shows a remarkable degree of

Received Sep. 11, 2019; revised Apr. 27, 2020; accepted May 13, 2020.

Author contributions: L.C. and C.V. designed research; L.C., P.S., E.V., B.T., and A.S. performed research; L.C., P.S., B.T., A.S., V.B., M.M.,T.B. and C.V. analyzed data; C.V., and L.C. wrote the paper.

This work was supported by Comitato Telethon Fondazione Onlus; contract grant number: GGP16131 to C. V, Regione Lombardia; contract grant number: "AMANDA" CUP_B42F16000440005 to C.V; Regione Lombardia Ne0n Progetto "Ne0n" POR-FESR 2014-2020, ID 239047, CUP E47F17000000009 to C.V. Else Kröner Foundation to T.B.

The authors declare no competing financial interests.

Correspondence should be addressed to Chiara Verpelli at chiara.verpelli@in.cnr.it.

https://doi.org/10.1523/JNEUROSCI.2194-19.2020

Copyright $(2020$ the authors cross-species similarity, suggesting highly conserved biological function (Blanchard et al., 2004; Minchin et al., 2008). Of note, SULT4A1 has an atypical enzymatic domain structure, as revealed by a recent crystal structure (Allali-Hassani et al., 2007), that may affect PAPS binding and substrate specificity: in fact, no sulfonation activity has been detected with potential sulfate donors or substrates (Falany et al., 2000; Allali-Hassani et al., 2007), suggesting that SULT4A1 may not exhibit classical catalytic activity in vivo or that the functional enzyme may be active as a component of a multienzyme complex (Falany et al., 2000). Thus, in the absence of any known substrate, the biological function of SULT4A1 remains unclear.

SULT4A1 tissue distribution has been examined in both humans and rodents and has been demonstrated to be predominantly expressed within the brain, although limited expression of mRNA and protein is detectable in other organs, such as kidney, lung, liver, and heart tissues (Alnouti et al., 2006; Sidharthan et al., 2014). In particular, the strongest protein expression 
has been detected in cerebral cortex, thalamus, cerebellum, and hippocampus (Liyou et al., 2003). In mouse, Sult4a1 mRNA expression is low in fetal brains and remains nearly unchanged until postnatal day 30 (P30), after which a marked increase in expression has been observed (Alnouti et al., 2006). Consistent with this, SULT4A1 protein expression was found to increase during neuronal differentiation (Idris et al., 2019). Notably, Sult4a1-KO (knock-out) mice display severe neurologic defects including tremor, rigidity, and seizure, and the survival of pups during postnatal development is strongly affected by the loss of Sult4a1 (Garcia et al., 2018).

A growing body of evidence supports SULT4A1 as a key player in neuronal maturation, and that the loss of SULT4A1 function can contribute toward neurodevelopmental disorders. For instance, SULT4A1 haploinsufficiency has been linked to neurologic symptoms of patients with Phelan-McDermid syndrome (PMS; Disciglio et al., 2014; Mitz et al., 2018; Ziats et al., 2019), with SULT4A1 deletion strongly correlatedwith a lower developmental quotient (Zwanenburg et al., 2016). In addition, single nucleotide polymorphisms in the SULT4A1 gene have been linked to schizophrenia susceptibility as well as to the severity of both psychotic and intellectual impairment, and antipsychotic treatment response (Brennan and Condra, 2005; Meltzer et al., 2008; Ramsey et al., 2011). Altogether, these findings suggest a role of SULT4A1 in neuronal development and function and in altered expression as a potential contributing factor in multiple neurodevelopmental disorders.

Despite this body of supporting literature, the role of SULT4A1 within neuronal development and function remains unassessed. Our results provide evidence that SULT4A1 has a pivotal role in the regulation of neuronal morphology and synaptic activity. Our data suggest that this effect is achieved through regulation, by direct protein-protein interaction, of the peptidylprolyl cis-trans isomerase Pin1 at the synaptic level. Pin1 is an enzyme that binds to phosphorylated serine/threonine-proline motifs and catalyzes cis-to-trans isomerization of prolines (Ranganathan et al., 1997; Shen et al., 1998). Notably acting to regulate synaptic strength through the regulation of postsynaptic scaffolds: for instance, in excitatory synapses Pin1 has been found to downregulate glutamatergic synaptic transmission by negatively modulating PSD-95/NMDAR (NMDA receptor) complex formation (Antonelli et al., 2016). Here we demonstrate that, by sequestering Pin1, SULT4A1 facilitates the formation of the PSD-95/ NMDAR complex in excitatory synapses, which is essential for NMDAR-mediated synaptic transmission and spine formation.

\section{Materials and Methods}

Constructs and virus generation. For RNA interference, an siRNA sequence targeting the Sult4a1 C terminus was designed following GenScript siRNA Target Finder instructions (GenScript). The following nucleotide sequence was used: AAGTGTGACCTCACGTTTGAC. The sequence was used to generate a short hairpin RNA (shRNA; indicated as shSult4a1 or shSULT), which was cloned into the pLVTHM-GFP vector (Wiznerowicz and Trono, 2003) using EcoRI and ClaI restriction sites. A scrambled form of shRNA was cloned into pLVTHM-GFP so as to generate the control shRNA (indicated as shCtrl) with the following nucleotide sequence: GCTGAGCGAAGGAGAGAT. Previously described pFlag-SULT4A1(Mitchell and Minchin, 2009) was used for the overexpression experiments alongside pLVTHM-GFP vector as an overexpression control. Site-directed mutagenesis was performed using the QuikChange Lightning Site-Directed Mutagenesis Kit (Agilent Technologies) to generate a construct resistant to interference by shSult4a1 (indicated as Sult4a1r or Sultr). In this construct, three nucleotides (G825A, T828C, C831T) of the shSult4a1 target site were altered without changing the amino acid sequence of the resultant protein.

For viral transduction, genetically modified lentiviruses were produced as previously described (Naldini et al., 1996; Lois et al., 2002), and the production was conducted with second- and third-generation lentiviral transfer vectors.

Animals. To prepare primary neuronal rat cultures, pregnant female rats (Rattus norvegicus) of the phylum Wistar strain were purchased from Charles River Laboratories. C57BL/6 wild-type mice were purchased from Charles River Laboratories. Mice and rats were housed under constant temperature $\left(22 \pm 1^{\circ} \mathrm{C}\right)$ and humidity $(50 \%)$ conditions with a $12 \mathrm{~h}$ light/dark cycle and were provided with food and water ad libitum. All experiments involving animals followed protocols in accordance with the guidelines established by the European Communities Council and the Italian Ministry of Health (Rome, Italy).

Cell lines and transfections. HEK293T cells were cultured at $37^{\circ} \mathrm{C}$ and $5 \% \mathrm{CO}_{2}$ atmosphere in DMEM (Thermo Fisher Scientific) supplemented with fetal bovine serum (10\%; Thermo Fisher Scientific), L-glutamine (2 mm; Euroclone), and PenStrep (1\%; Thermo Fisher Scientific). Cells were transfected using Lipofectamine 2000 (Thermo Fisher Scientific) and collected 24-48 h after transfection.

Primary neuronal cell culture. Low-density rat cortical neuronal cultures were prepared from embryonic day 18 (E18) rat embryos (Charles River Laboratories) as previously described (Verpelli et al., 2010). Neurons were plated at $150-200$ cells $/ \mathrm{mm}^{2}$ density on 6- or 12 -well plates (Euroclone) coated with $0.01 \mathrm{mg} / \mathrm{ml}$ poly-L-lysine (SigmaAldrich). Neurons were cultured in Neurobasal Medium (Thermo Fisher Scientific) supplemented with the previously described B27 Medium (Chen et al., 2008). Cells were cultured on 6- and 12-well plates for protein biochemical analysis, whereas 12 -well plates with acid-treated coverslips (VWR) were used for immunocytochemical or electrophysiological analysis. At day in vitro 7 (DIV7), neurons were lentivirally transduced or transfected using Lipofectamine 2000 (Thermo Fisher Scientific). Biochemical, electrophysiological, and morphologic experiments were performed at DIV14.

Diethyl-1,3,6,8-tetrahydro-1,3,6,8-tetraoxobenzol-phenanthroline-2,7diacetate treatment. To inhibit Pin1 catalytic activity, cortical neurons were treated with $\mathrm{PiB}$ (diethyl-1,3,6,8-tetrahydro-1,3,6,8-tetraoxobenzolphenanthroline-2,7-diacetate). PiB (Calbiochem) was resuspended in DMSO. As acute treatment, neurons were treated for $48 \mathrm{~h}$ with $2.5 \mu \mathrm{M}$ $\mathrm{PiB}$ and analyzed at DIV14; as chronic treatment, neurons were treated with $1 \mu \mathrm{M}$ PiB every other day, from DIV7 to DIV14.

DTM treatment. DTM (Calbiochem), the pharmacological inhibitor of Pin 1 catalytic activity, was resuspended in DMSO. As acute treatment, cortical neurons were treated for $48 \mathrm{~h}$ with $4 \mu \mathrm{M}$ DTM and analyzed at DIV14.

Derivation of human induced pluripotent stem cells and neuronal differentiation. Human blood samples were collected according to a clinical protocol approved by the local bioethical committees of different medical centers. Participating individuals have been informed of the objectives of the study and signed an informed consent form before inclusion in the study. Peripheral blood mononuclear cells (PBMCs) were isolated using Ficoll and grown in StemPro-34 SFM Medium (Thermo Fisher Scientific), supplemented with L-glutamine (2 mM; Euroclone), PenStrep (1\%; Thermo Fisher Scientific), SCF $(100 \mathrm{ng} / \mathrm{ml}$; Thermo Fisher Scientific), FLT-3 (100 ng/ml; Thermo Fisher Scientific), IL-3 (20 ng/ml; Thermo Fisher Scientific), and IL-6 (20 ng/ml; Thermo Fisher Scientific). To generate human induced pluripotent stem cells (iPSCs), PBMCs were transduced with 2.0 Sendai virus particles containing four Yamanaka factors using the CytoTune-iPS Sendai Reprogramming Kit (Thermo Fisher Scientific). After $7 \mathrm{~d}$, transduced cells were plated on culture dishes coated with hESC-qualified Matrigel (Corning) and grown in feeder-free conditions with Essential 8 Medium (Thermo Fisher Scientific). Three to 4 weeks after transduction, iPSC colonies were manually picked for further expansion or analysis.

For neural stem cells (NSCs) derivation, iPSCs were detached with UltraPure EDTA (Thermo Fisher Scientific) and plated on Matrigelcoated 6-well plates in Essential 8 medium. After $24 \mathrm{~h}$, the spent medium was replaced with PSC Neural Induction Medium (Thermo Fisher 
Scientific) and subsequently was changed every other day following manufacturer instructions. On day 7 of neuronal induction, NSCs (P0) were harvested with StemPro Accutase (Thermo Fisher Scientific) and plated on Matrigel-coated plates for further expansion.

To obtain terminally differentiated neurons, proliferating NSCs were plated on Matrigel-coated 6- or 12-well plates and cultured in Neurobasal Medium supplemented with B27 without vitamin A (2\%; Thermo Fisher Scientific), PenStrep (1\%; Thermo Fisher Scientific), Glutamax (2 mm; Thermo Fisher Scientific), NT-3 (10 ng/ml; Miltenyi Biotec), BDNF (10 ng/ml; Miltenyi Biotec), GDNF (10 ng/ml; Miltenyi Biotec), retinoic acid ( $1 \mu \mathrm{M}$; Sigma-Aldrich), and growth for at least $40 \mathrm{~d}$ (Borroni et al., 2017). Half media changes were performed every 2-3 d thereafter.

Biochemical analysis. Cells or brain were lysed in prechilled buffered sucrose [0.32 м sucrose (Sigma-Aldrich)/4 mm HEPES-NaOH buffer (Sigma-Aldrich), pH 7.3, protease inhibitors (Sigma-Aldrich), phosphatase inhibitors (Roche)] and analyzed via Bradford Protein Assay (BioRad) to assess protein concentration. For total lysate, proteins were directly solubilized in $4 \times$ loading buffer $(250 \mathrm{~mm}$ Tris, $40 \%$ glycerol, $0.008 \%$ bromophenol blue; all from Sigma-Aldrich). In other cases, fractionation took place before solubilization to obtain a synaptosomeenriched fraction (P2), as previously published (Vicidomini et al., 2017).

For immunoblotting, primary antibodies and HRP-conjugated secondary antibodies were applied overnight $(\mathrm{o} / \mathrm{n})$ and for $1 \mathrm{~h}$, respectively. Chemiluminescence was induced using an ECL Kit (GE Healthcare), and densitometry was performed using Fiji/ImageJ (US National Institutes of Health).

Immunoprecipitation assay. To evaluate the interaction among Pin1, SULT4A1, and PSD-95; and primary neurons were lysed in NP-40 lysis buffer (1\% NP-40, $50 \mathrm{~mm}$ Tris, $\mathrm{pH}$ 8.0, $150 \mathrm{~mm} \mathrm{NaCl}$, and protease inhibitor cocktail; all from Sigma-Aldrich) and subjected to synaptosomal fractionation, as previously described (Vicidomini et al., 2017). P2 fractions were precleared in NP-40 buffer for $4 \mathrm{~h}$ and then incubated o/n at $4^{\circ} \mathrm{C}$ with protein A-Sepharose beads (GE Healthcare) conjugated to $10 \mu \mathrm{g} / \mathrm{ml}$ anti-Pin1 antibody (Santa Cruz Biotechnology) or matched control IgG (Sigma-Aldrich). The beads were then washed with $0.5 \%$ NP-40 buffer, resuspended in loading buffer, warmed at $65^{\circ} \mathrm{C}$ for $10 \mathrm{~min}$, and analyzed using SDS-PAGE. Samples of precleared P2 fractions were collected before immunoprecipitation and used as input signals.

Electrophysiological recordings. Whole-cell patch-clamp recordings in voltage-clamp configuration were performed at room temperature $\left(\sim 25^{\circ} \mathrm{C}\right)$ on DIV14 neurons using a Multiclamp 700A Amplifier and pClamp 10.5 software (Molecular Devices). Signals were filtered at $1 \mathrm{kHz}$ and sampled at $10 \mathrm{kHz}$. Postsynaptic currents were recorded at $-70 \mathrm{mV}$ in an external bath solution containing the following (mM): $129 \mathrm{NaCl}$, $1.25 \mathrm{NaH}_{2} \mathrm{PO}_{4}, 35$ glucose, $1.8 \mathrm{MgSO}_{4}, 1.6 \mathrm{CaCl}_{2}, 3 \mathrm{KCl}$, and $10 \mathrm{HEPES}$, $\mathrm{pH}$ 7.4, with $\mathrm{NaOH}$. Spontaneous EPSCs (sEPSCs) were recorded in the presence of gabazine $(125 \mu \mathrm{M})$ with an intracellular pipette solution containing the following (mM): $120 \mathrm{~K}$-gluconate, $15 \mathrm{KCl}, 2 \mathrm{MgCl}_{2}$,

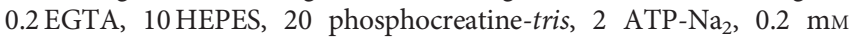
GTP-Na $2,0.1$ leupeptin, and 3 lidocaine $N$-ethyl bromide, $\mathrm{pH} 7.2$, with $\mathrm{KOH}$. Spontaneous IPSCs (sIPSCs) were recorded in the presence of kynurenic acid $(3 \mathrm{mM})$ with an intracellular pipette solution containing the following (mM): $135 \mathrm{CsCl}, 2 \mathrm{MgCl}_{2}$, 0.2 EGTA, 10 HEPES, 20 phosphocreatine-tris, 2 ATP-Na $2,0.2$ mM GTP-Na, 0.1 leupeptin, and 3 lidocaine $\mathrm{N}$-ethyl bromide, $\mathrm{pH} 7.2$, with $\mathrm{KOH}$; in these conditions, the $\mathrm{Cl}^{-}$ reversal potential was $0 \mathrm{mV}$, thus allowing recording at hyperpolarized potentials $(-70 \mathrm{mV})$. Signals were filtered at $1 \mathrm{kHz}$ and sampled at $10 \mathrm{kHz}$. The quantification of the instantaneous frequency, the inverse of the interevent interval, and of the amplitude of postsynaptic currents was performed on 2-min-long recordings with Clampfit 10.5 using a threshold search (threshold set at four times the baseline root mean square noise).

NMDA-mediated currents $\left(I_{\mathrm{NMDA}} \mathrm{s}\right)$ were elicited by the perfusion of $100 \mu \mathrm{m}$ NMDA and recorded at $-70 \mathrm{mV}$ with an external bath solution containing the following (mM): $150 \mathrm{NaCl}$, three $\mathrm{KCl}, 3 \mathrm{CaCl}_{2}, 10 \mathrm{HEPES}$, 8 glucose, $0.1 \mathrm{D}$-serine, 0.001 tetrodotoxin, $0.01 \mathrm{DNQX}, 0.01$ picrotoxin, and 0.001 CGP 55845, pH 7.4 with $\mathrm{NaOH}$. AMPA-mediated currents
$\left(I_{\mathrm{AMPA}} \mathrm{s}\right)$ were elicited by the perfusion of $100 \mu \mathrm{M}$ AMPA and recorded at $-70 \mathrm{mV}$ with an external bath solution containing the following (mM): $150 \mathrm{NaCl}, 3 \mathrm{KCl}, 3 \mathrm{CaCl}_{2}, 2 \mathrm{MgCl}_{2}, 10 \mathrm{HEPES}, 8$ glucose, 0.1 and 0.001 tetrodotoxin, $0.01 \mathrm{D}$-CPP, 0.01 picrotoxin, and 0.001 CGP 55845, $\mathrm{pH} 7.4$ with $\mathrm{NaOH}$. The internal pipette solution contained the following (mM): $135 \mathrm{CH}_{3} \mathrm{O}_{3} \mathrm{SCs}, 8 \mathrm{NaCl}$, 0.5 EGTA, 10 HEPES, 0.3 GTP-Na ${ }_{2}$, and 4 ATP-Mg, pH 7.2 with CsOH. Application of NMDA and AMPA was performed with a local fast perfusion system controlled by electronic valves (RSC-200 Rapid Solution Changer, Bio-Logic Science Instruments); the tip of a multichannel manifold was placed at a distance of $0.2 \mathrm{~mm}$ from the patched neuron, allowing a fast solution exchange. To avoid drug accumulation, $10 \mathrm{~min}$ of washout was applied after each recording. Peak $I_{\mathrm{NMDA}}$ and $I_{\mathrm{AMPA}}$ values were normalized to the membrane capacitance to obtain the current densities for statistical analysis.

In utero electroporation, section preparation, and immunohistochemistry. In utero electroporation was used to deliver shCtrl- and shSult4a1expressing vectors to the ventricular RGCs of CD1 mouse embryos as previously described (Sessa et al., 2008). Briefly, uterine horns of E13.5 pregnant dams were exposed by midline laparotomy after anesthetization with Avertin $(312 \mathrm{mg} / \mathrm{kg}$; Sigma-Aldrich). One microliter of DNA plasmids corresponding to $3 \mu \mathrm{g}$ mixed with $0.03 \%$ fast-green dye in PBS was injected in the telencephalic vesicle using a pulled micropipette through the uterine wall and amniotic sac. Seven millimeter platinum tweezer-style electrodes were placed outside the uterus over the telencephalon and five pulses of $40 \mathrm{~V}, 50 \mathrm{~ms}$ in length, were applied at $950 \mathrm{~ms}$ intervals by using a BTX square wave electroporator. The uterus was then replaced in the abdomen, the cavity was filled with warm sterile PBS, and the abdominal muscle and skin incisions were closed with silk sutures.

At P30, GFP-positive brains were dissected for subsequent analyses. To this purpose, animals were anesthetized with an intraperitoneal injection of Avertin and perfused with 5\% sucrose and 4\% paraformaldehyde. Then, brains were left overnight in $4 \%$ paraformaldehyde, followed by incubation in 30\% sucrose. Finally, brains were included in cryomolds with Tissue-Tek OCT compound (Sakura) and stored at $-80^{\circ} \mathrm{C}$ until cryostat sectioning. The 5 - $\mu \mathrm{m}$-thick slices were collected on polysine microscope adhesion slides (Thermo Fisher Scientific) and then incubated in blocking solution (3\% BSA, $10 \%$ goat serum, $0.4 \%$ Triton $\mathrm{X}$ 100 , diluted in PBS). Primary and fluorophore-conjugated secondary antibodies were diluted in blocking solution and applied respectively o/n at $4^{\circ} \mathrm{C}$ and $1 \mathrm{~h}$ at room temperature. Glass coverslips were mounted on slides with Mounting Medium (VectaShield).

All procedures were approved by the Italian Ministry of Health and the San Raffaele Scientific Institute Animal Care and Use Committee in accordance with the relevant guidelines and regulations.

Immunocytochemistry. Cells were fixed in $4 \%$ paraformaldehyde, $4 \%$ sucrose in PBS (136.8 $\mathrm{mm} \mathrm{NaCl}, 2.68 \mathrm{~mm} \mathrm{KCl}, 10.1 \mathrm{~mm} \mathrm{Na}_{2} \mathrm{HPO}_{4}$, and $1.76 \mathrm{~mm} \mathrm{KH}_{2} \mathrm{PO}_{4}$, $\mathrm{pH} 7.4$; all from Sigma-Aldrich) at room temperature for $10 \mathrm{~min}$. For colocalization analysis, neurons were fixed in $4 \%$ paraformaldehyde, $4 \%$ sucrose in PBS at room temperature for $5 \mathrm{~min}$, and then in cold methanol at $-20^{\circ} \mathrm{C}$ for $10 \mathrm{~min}$. Primary antibodies were diluted in homemade gelatin detergent buffer (GDB; $30 \mathrm{~mm}$ phosphate buffer, pH 7.4, $0.2 \%$ gelatin, $0.5 \%$ Triton X-100, $0.8 \mathrm{M} \mathrm{NaCl}$; all SigmaAldrich) and applied $\mathrm{o} / \mathrm{n}$ at $4^{\circ} \mathrm{C}$. Secondary antibodies conjugate with fluorophores (Jackson ImmunoResearch) were also diluted in GDB buffer and applied for $1 \mathrm{~h}$. DAPI staining (Thermo Fisher Scientific) was conducted at a final concentration of $0.5 \mu \mathrm{g} / \mathrm{ml}$. Coverslips were mounted on precleaned microscope slides using Mowiol mounting medium (Osborn and Weber, 1982).

Image acquisition and processing. Confocal images were obtained using an LSM 510 Meta Confocal Microscope (Carl Zeiss) with Zeiss $63 \times, 40 \times$, or $20 \times$ objectives at a resolution of $1024 \times 1024$ pixels. Images represent averaged intensity Z-series projections of two to seven individual images taken at depth intervals of $\sim 0.45 \mu \mathrm{m}$.

Secondary and tertiary dendrites with comparable length and width were selected for dendritic spine analyses. Dendritic spine analysis was performed as described in the study by Verpelli et al. (2011) but using Fiji/ImageJ software (US National Institutes of Health) for the quantification. 
For colocalization analysis, measurements were performed using MetaMorph software (Universal Imaging). For neuronal arborization analysis, primary and secondary dendrites were measured manually after identifying the points of intersection. The dendrites originating from the cell body were considered as primary dendrites, while all the dendrites originating from the primary dendrites at the point of intersection were considered as secondary dendrites. Sholl analysis and total dendrites length quantification were performed using Fiji/ImageJ software. Branching point intersections were counted and plotted against distance from the soma.

Antibodies. The following primary antibodies were used: mouse anti$\beta$-actin (catalog \#A5316, Sigma-Aldrich), mouse anti- $\beta$ III-tubulin (catalog \#MAB1637, Sigma-Aldrich), mouse anti-GABA ${ }_{\mathrm{A}} \mathrm{R} \alpha 1$ (catalog \#75-136, NeuroMab), mouse anti-GAD65 (catalog \#198111, Synaptic System), mouse anti-GAD67 (catalog \#sc-28376, Santa Cruz Biotechnology), mouse anti-GFP (catalog \#11814460001, Roche), rabbit anti-GluA1 (catalog \#AB1504, Millipore), mouse anti-GluA2 (catalog \#75-002, NeuroMab), mouse anti-GluN1 (catalog \#556308, BD), mouse anti-GluN2A (catalog \#75-288, NeuroMab), rabbit anti-GluN2B (cata$\log \# 06-600$, Millipore), mouse anti-MAP2 (catalog \#ab11268, Abcam), rabbit anti-mGlu5 (catalog \#AB5675, Millipore), mouse anti-Nestin (catalog \#MAB5326, Millipore), mouse anti-NLGN1 (catalog \#75-160, NeuroMab), mouse anti-Oct4 (catalog \#sc-5279, Santa Cruz Biotechnology), mouse anti-Pin1 (catalog \#sc-46660, Santa Cruz Biotechnology), mouse anti-PSD-95 (catalog \#75-028, NeuroMab), rabbit anti-PSD-95 (catalog \#D27E11, Cell Signaling Technology), rabbit antiSox2 (catalog \#11064-1-AP, Proteintech), rabbit anti-SULT4A1 (catalog \#12578-1-AP, Proteintech), and rabbit anti-VGAT (catalog \#131003, Synaptic System).

All HRP- and fluorophore-conjugated secondary antibodies were purchased from Jackson ImmunoResearch.

Experimental design and statistical analysis. Data are expressed as the mean \pm SEM or percentage, analyzed for statistical significance, and displayed by Prism 7 software (GraphPad Software). Shapiro-Wilk test or D'Agostino-Pearson tests were applied to test the normal distribution of experimental data. Normal distributions were compared with $t$ test or ANOVA with appropriate post hoc test. Non-normal distributions were compared with the nonparametric Mann-Whitney test or KruskalWallis test with appropriate post hoc test, as indicated. The accepted level of significance was $p \leq 0.05$.

Statistical analyses for electrophysiological experiments were performed with OriginPro software. Distributions of cumulative probabilities were compared with the Kolmogorov-Smirnov test.

No statistical methods were used to predetermine sample sizes, but sample sizes were chosen to be similar to those reported in previous publications.

In the figures, we also show the mean as an estimator of central tendency when we have used a nonparametric test, for consistency with other figures in the article and because it is more intuitive to compare the mean values.

\section{Results}

\section{Sulfotransferase 4A1 expression is increased during brain maturation}

SULT4A1 is a brain-specific sulfotransferase highly conserved among vertebrate that appears to have a particularly strong expression in distinct areas of the cerebral cortex (e.g., prefrontal cortex), cerebellum (e.g., neuronal stroma), and brainstem (e.g., hypoglossal nucleus; Liyou et al., 2003). To investigate SULT4A1 expression during neuronal maturation, we analyzed protein lysates harvested from rat cortical neurons at DIV1, DIV7, and DIV14. SULT4A1 expression was almost undetectable at DIV1 and increased during the neuronal maturation (Fig. 1A; DIV1, $0.03 \pm 0.01 ;$ DIV7, $0.18 \pm 0.02 ;$ DIV14, $0.38 \pm 0.03)$. Then, SULT4A1 expression was evaluated in vivo during mouse brain development. Western blot (WB) analysis showed that SULT4A1 protein levels increased from P0 to P30. Moreover, high levels of
SULT4A1 were also maintained in adult mice (P60), suggesting an important role of SULT4A1 both during brain maturation and adulthood (Fig. 1B; P0, $0.12 \pm 0.03$; P7, $0.48 \pm 0.09$; P14, $0.66 \pm 0.11 ; \mathrm{P} 21,0.91 \pm 0.23 ; \mathrm{P} 30,0.79 \pm 0.15 ; \mathrm{P} 60,1.0 \pm 0.3$ ). SULT4A1 is known to have particularly strong expression in the brain but, to date, the current knowledge of SULT4A1 area-specific expression is restricted to human and rat tissues (Liyou et al., 2003). To assess the tissue distribution in adult mouse brain, WB analysis of total lysates of hippocampus $(\mathrm{H})$, striatum $(\mathrm{S})$, cerebral cortex $(\mathrm{Cx})$, and cerebellum $(\mathrm{Cb})$ was performed on 2 month-old male mice. SULT4A1 was found to be highly expressed in all analyzed areas (Fig. $1 C ; \mathrm{H}, 0.71 \pm 0.05$; $\mathrm{S}$, $0.93 \pm 0.06 ; \mathrm{Cx}, 1.19 \pm 0.03 ; \mathrm{Cb}, 1.33 \pm 0.10)$. Finally, no significant difference in area-specific protein expression was observed between male and female animals [Fig. $1 D ; \mathrm{H}$ : male (M), $0.69 \pm 0.12$; female $(\mathrm{F}), 0.67 \pm 0.12 ; \mathrm{S}$ : $\mathrm{M}, \quad 0.80 \pm 0.12 ; \mathrm{F}$, $0.85 \pm 0.13$; Cx: M, $0.87 \pm 0.10 ; \mathrm{F}, 1.05 \pm 0.14$; Cb: M, $1.16 \pm$ $0.20 ; \mathrm{F}, 1.07 \pm 0.24]$. The immunostaining of cortical neurons revealed a localization of SULT4A1 to neuronal cell bodies and dendrites (Fig. 1E).

To determine SULT4A1 protein expression in human neurons, human iPSCs were differentiated into Nestin-positive NSCs and subsequently into neurons (Fig. $1 F$ ), and were analyzed for SULT4A1 protein levels during differentiation. Human SULT4A1 expression was found to be increased during differentiation from NSCs (day 0) to mature neurons (day 40; Fig. $1 G$; day $0,0.23 \pm 0.08$; day $10,0.14 \pm 0.06$; day $20,0.63 \pm 0.02$; day $30,1.57 \pm 0.1$; day $40,2.04 \pm 0.17)$.

\section{SULT4A1 silencing reduces neuronal arborization and dendritic spine density}

To evaluate the impact of SULT4A1 on neuronal morphogenesis, specific shRNAs for Sult4a1 (shSult4a1) were designed and validated in Sult4a1-transfected HEK cells (Extended Data Fig. 2$1 A)$. shRNA 1 was chosen for further analysis because of its stronger effect on Sult4a1 protein level, and therefore indicated as shSult4a1. To confirm the specificity of shSult4a1 silencing activity, shSult4a1 was expressed together with a construct resistant to interference (Sult4a1r) and restoration of the Sult4a1 protein level was verified (Extended Data Fig. 2-1B).

To assess the role of SULT4A1 in early neuronal development, cortical neurons were transfected at DIV7 with shSult4a1 or control scrambled shRNA (i.e., shCtrl; Fig. 2A). Dendrite morphology of transfected neurons was then evaluated by Sholl analysis at DIV14 (Fig. 2B). Sult4a1 silencing resulted in a simplification of neuronal branching, indicated by the reduction of branching points, primary dendrites, secondary dendrites, and total dendrites length (Fig. $2 B-E$ ). These morphologic alterations were prevented by cotransfection of shSult4al with Sult4alr (Fig. $2 A-E$; branching points: condition factor, $p<0.0001$; condition by distance interaction, $p<0.0001$; primary dendrites: shCtrl, $7.30 \pm 0.39$; shSult4a1, $4.70 \pm 0.43$; Sult4a1r, $7.3 \pm 0.45$; secondary dendrites: shCtrl, $11.60 \pm 0.94$; shSult4a1, $6.26 \pm 0.51$; Sult4a1r, $9.55 \pm 0.65$; total dendrites length: shCtrl, $1430.21 \pm$ 132.93; shSult4a1, 644.78 \pm 61.86; Sult4a1r, $1429.04 \pm 140.90)$.

The analysis of dendritic spine morphology revealed that Sult4a1 knocked-down neurons exhibit a significant decrease in spines number (Fig. 2I; spine numbers: shCtrl, $4.07 \pm 0.18$; shSult4a1, $2.96 \pm 0.24$; Sult4a1r, $4.05 \pm 0.36$ ), but the morphology of the remaining spines is unchanged (Fig. 2G,H; spine length: shCtrl, $1.97 \pm 0.10 ;$ shSult4a1, $1.90 \pm 0.10 ;$ Sult4a1r, $1.86 \pm 0.09$; spines width: shCtrl, $1.03 \pm 0.05$; shSult4a1, $1.04 \pm$ 0.05 ; Sult4a1r, $1 \pm 0.03$ ). A slight increase of the number of 
A

\begin{aligned} & \multicolumn{2}{c}{ Day-in-vitro } \\ \cline { 2 - 2 } & $1 \quad 7 \quad 14 \\$ SULT4A1 & \\ PSD-95 & \\ Actin & \end{aligned}

C

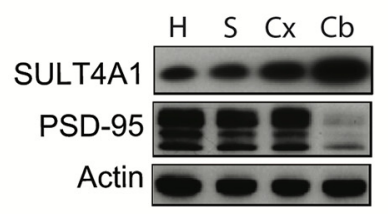

E

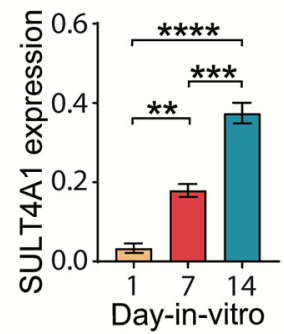

B

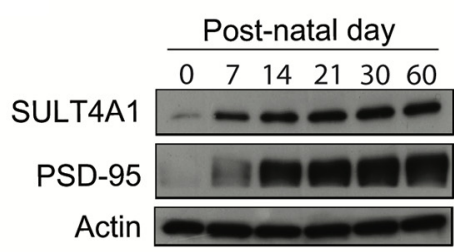

D

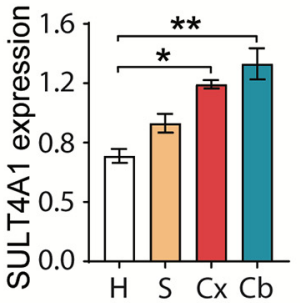

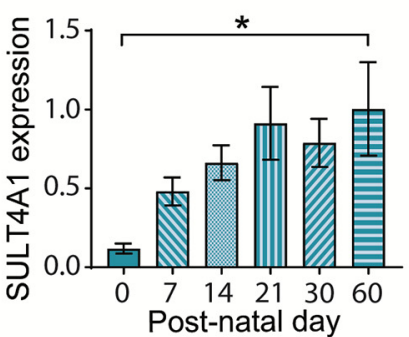

Post-natal day

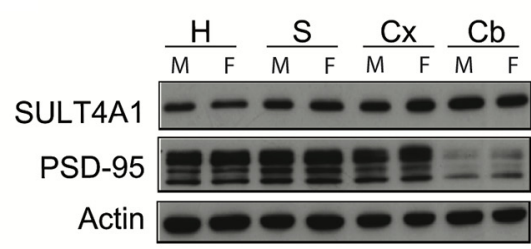

F

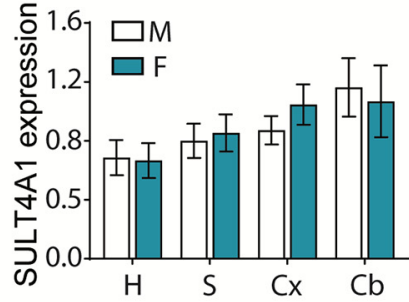

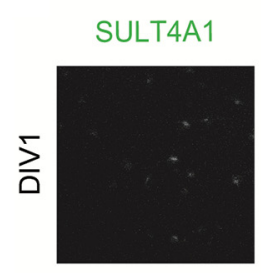
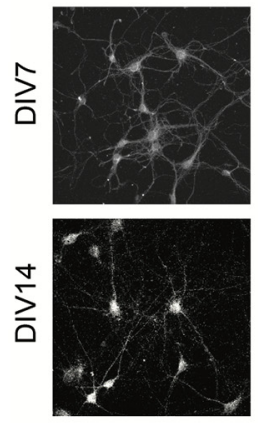
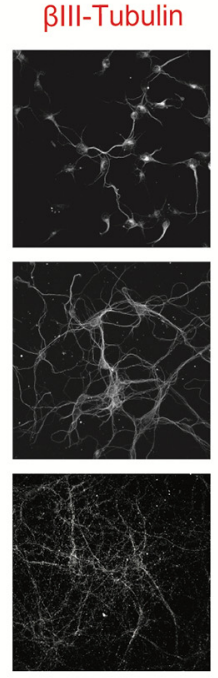
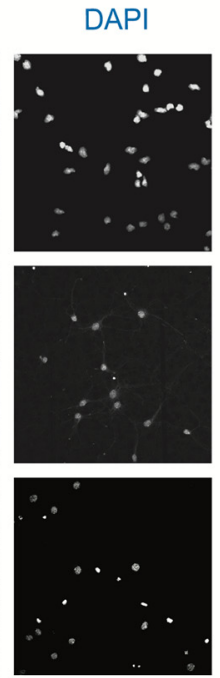
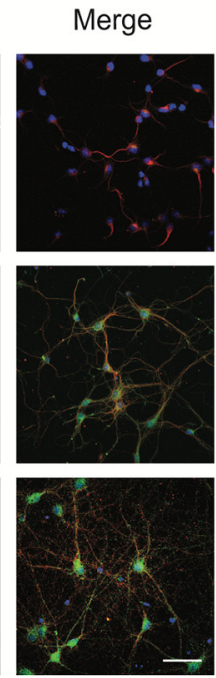

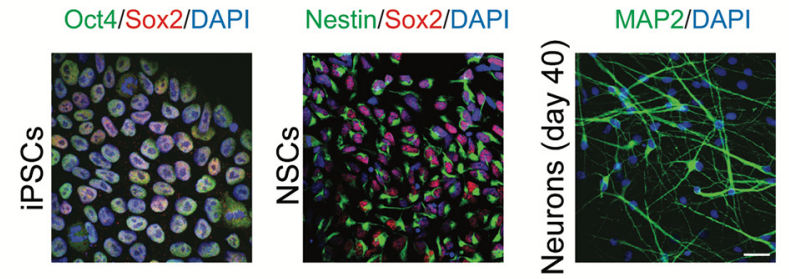

G
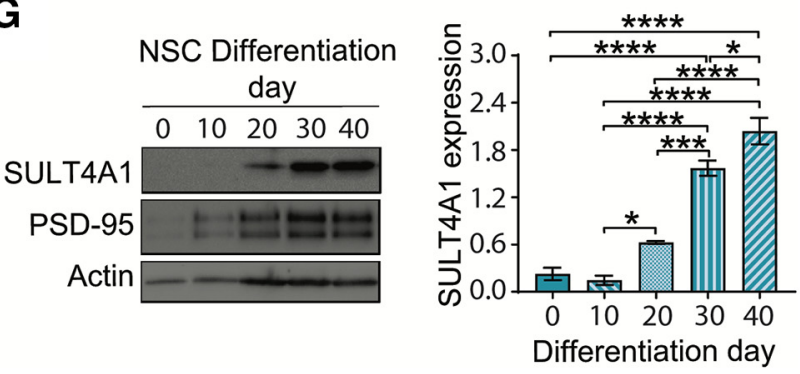

Figure 1. Physiologic expression of SULT4A1 protein during neuronal development. $A$, Representative Western blots of total cell lysates derived from rat cortical neurons at different days in vitro ( $n=4$ independent cultures for all conditions; one-way ANOVA test, $p<0.0001$; Tukey's post hoc test, ${ }^{* *} p=0.0011,{ }^{* * *} p=0.0001,{ }^{* * * *} p<0.0001$ ). $\boldsymbol{B}$, Biochemical analysis of total brain lysates derived from wild-type male mice at different postnatal days ( $n=3$ animals for all conditions). $C$, Representative Western blots of protein lysates from $H, S, C x$, and $C b$ derived from adult (P60) wild-type mice ( $n=4$ animals for all conditions; Kruskal-Wallis test, $p<0.0001$; Dunn's post hoc test, $\left.{ }^{*} p=0.0451 ;{ }^{* *} p=0.0065\right)$. D, Area-specific expression of SULT4A1 was also compared between adult $\mathrm{M}$ and $\mathrm{F}$ mice ( $n=3$ animals for all conditions; two-way ANOVA, $p=0.8362$; Sidak's post hoc test). $\boldsymbol{E}$, Representative immunocytochemical staining for SULT4A1 (green), $\beta$ III-Tubulin (red), and DAPI (blue), in rat cortical neurons at DIV1, DIV7, and DIV14. Scale bar, $50 \mu \mathrm{m}$. $\boldsymbol{F}$, Left, Representative immunocytochemical staining of 0ct4 (green), Sox2 (red), and DAPI (blue) of iPSCs obtained from healthy control individuals. Middle, iPSC-derived NSCs stained for Nestin (green), Sox2 (red), and DAPI (blue). Right, Representative immunostaining for MAP2 (green) and DAPI (blue) of NSC-derived neurons after $40 \mathrm{~d}$ of differentiation. Scale bar, $50 \mu \mathrm{m}$. G, Representative immunoblots of total cell lysates obtained from human neurons, sampled during differentiation from day 0 (NSC stage) until day 40 (mature neuron stage; $n=3$ independent cultures for all conditions; one-way ANOVA, $p<0.0001 ;$ Tukey's post hoc test: 0 vs $30, p<0.0001 ; 0$ vs $40, p<0.0001 ; 10$ vs $20, p=0.0371 ; 10$ vs $30, p<0.0001 ; 10$ vs $40, p<0.0001 ; 20$ vs $30, p=0.0003 ; 20$ vs $40, p<0.0001 ; 30$ vs $40, p=0.0411$ ). Data represent the mean \pm SEM.

spines with filopodia-like morphology was observed in Sult4a1 knocked-down neurons (Fig. 2J). Cotransfection of shSult4a1 with Sult4a1r prevented these morphologic alterations (Fig. 2A-J).

To further confirm the role of SULT4A1 in modulating dendritic arborization and spine morphology in vivo, in utero electroporation was performed on wild-type mouse cortical neurons with shSult4a1 or shCtrl. Brain slices from 1-month-old electroporated animals were analyzed by confocal microscopy to measure dendritic complexity (Fig. $2 K$ ). Sholl analysis showed that, as in vitro, the number of branching points is decreased in cortical neurons following Sult4a1 knockdown (Fig. $2 L$; condition factor, $p=0.0112$; condition by distance interaction, $p=0.0629$ ). Moreover, Sult4a1 knockdown caused a significant reduction of total dendrite length (Fig. $2 M$; shCtrl, $421.4 \pm 28.35$; shSult4al, $292.86 \pm 40.42$ ).

The reduction in spine number was confirmed, in vivo, in shSult4a1 electroporated cortical neurons (Fig. $2 N-R$; spines numbers: shCtrl, $12 \pm 0.37$; shSult4a1, $10.81 \pm 0.5$; spine length: shCtrl, $1.23 \pm 0.12$; shSult4a1, $1.33 \pm 0.04$; spine width: shCtrl,

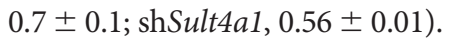

To assess the role of SULTA4A1 dosage in modulating neuronal morphology, neurons were transfected at DIV7 with GFP or GFP plus Sult4a1 and stained at DIV14 with anti-SULT4A1 
A
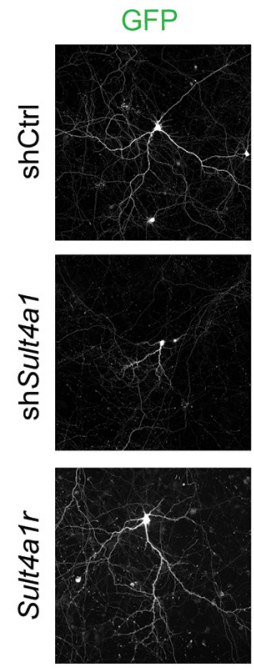
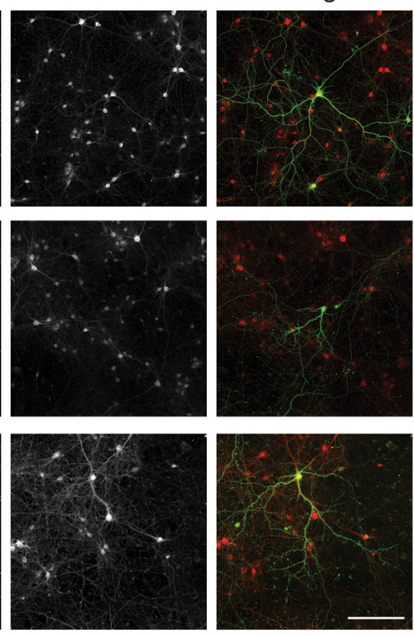

F

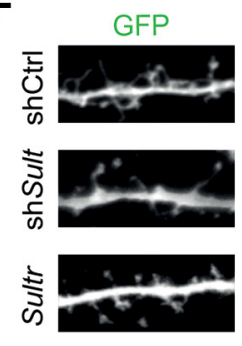

SULT4A1
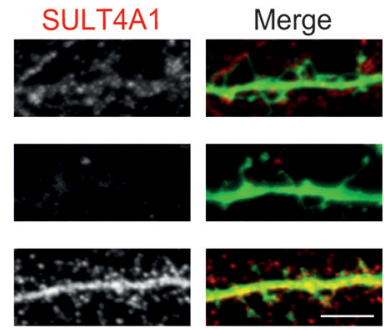

G

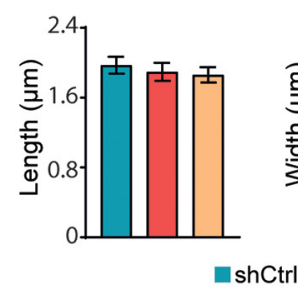

B

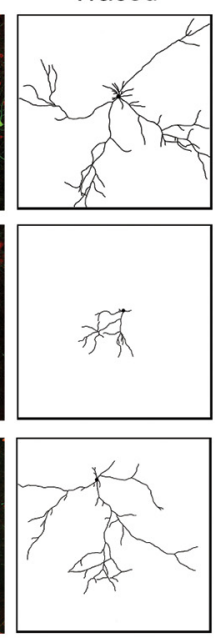

C

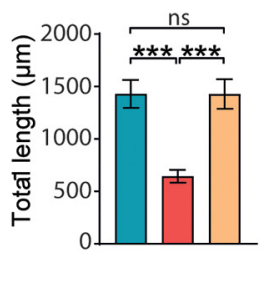

D

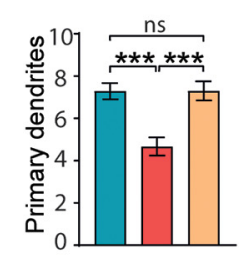

E

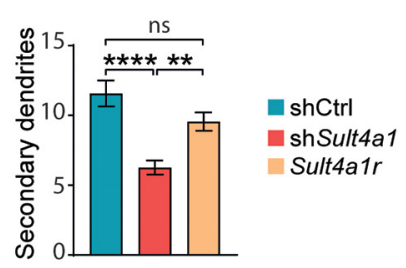

K

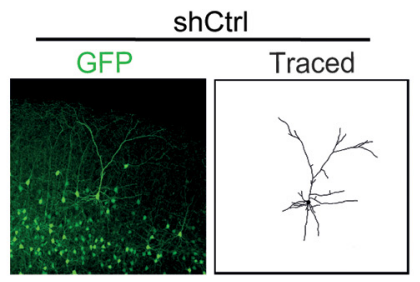

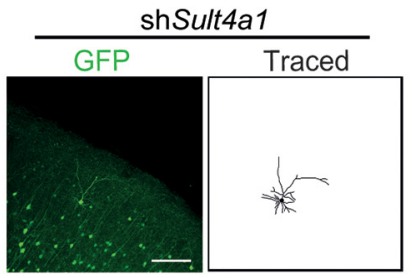

H

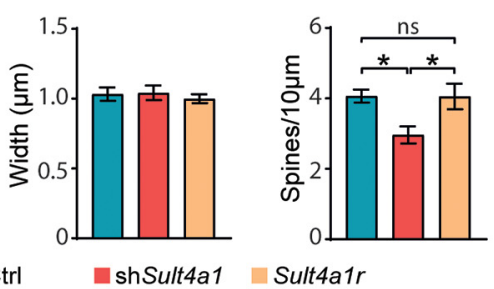

J
L

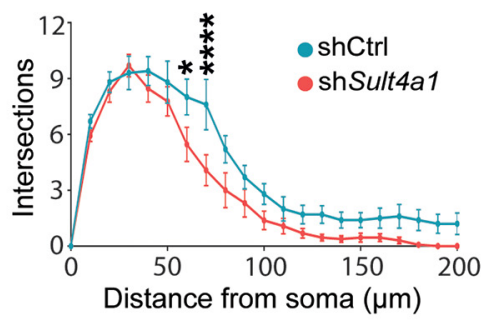

M

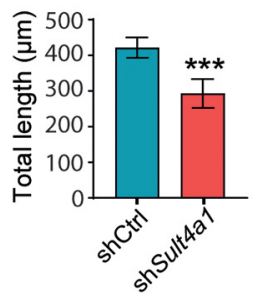

N

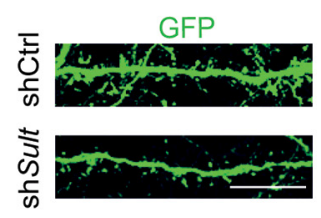

0

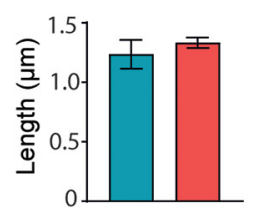

P

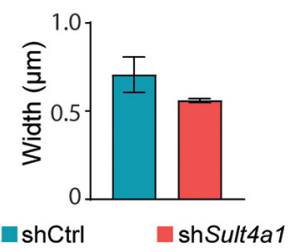

Q

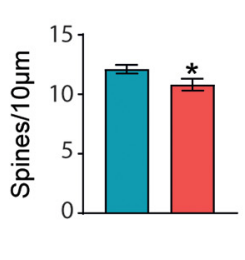

R

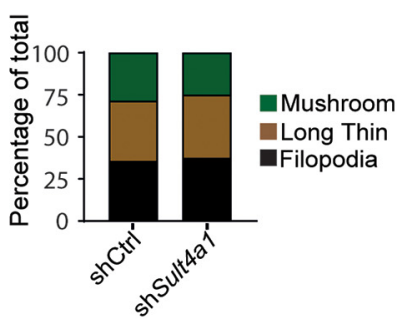

Figure 2. Sult4a1 silencing reduces neuronal branching and dendritic spine density. $A$, Representative immunofluorescence images and relative traces of rat cortical neurons transfected with the scrambled shRNA (sh(trl), the Sult4a1-specific shRNA (shSult4a1), or shSult4a1 together with the resistant construct (Sult4a1r). Neurons were stained for SULT4A1 (red) to assess protein expression. Scale bar, $100 \mu \mathrm{m}$. B. Results of Sholl analysis and quantification of the number of intersections, plotted against the distance from the soma (shCtrl, $n=13$ individual neurons; shSult4a1, $n=15$ individual neurons; Sult4a1r, $n=19$ individual neurons; two-way ANOVA, $p<0.0001$; Tukey's post hoc test, *shSult4a1 vs shCtrl; \#shSult4a1 vs Sult4a1r; $30 \mu$ m:

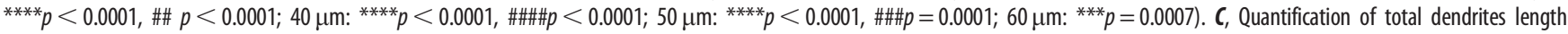
(shCtrl, $n=10$ individual neurons; shSult4a1, $n=13$ individual neurons; Sult4a1r, $n=13$ individual neurons; one-way ANOVA, $p<0.0001$; Tukey's post hoc test, shCtrl vs shSult4a1, $p=0.0001$; shSult4a1 vs Sult4a1r, $p=0.0001$; ns, not significant). $\boldsymbol{D}-\boldsymbol{E}$, Quantification of the number of primary (D) and secondary $(\boldsymbol{E})$ dendrites [shCtrl, $n=21$ individual neurons; shSult4a1, $n=18$ individual neurons; Sult4a1r, $n=20$ individual neurons; $\boldsymbol{D}$ : one-way ANOVA, $p<0.0001$; Tukey's post hoc test: shCtrl vs shSult4a1, $p=0.0002$; shSult4a1 vs Sult4a1r, $p=0.0002$; ns, not significant; $\boldsymbol{E}$ : one-way ANOVA, $p<0.0001$; Tukey's post hoc test, shCtrl vs shSult4a1, $p<0.0001$; shSult4a1 vs Sult4a1r, $p=0.0079$; ns, not significant]. $\boldsymbol{F}$, Representative immunofluorescence images showing dendritic spines of rat cortical neurons transfected with shCtrl, shSult4a1, or shSult4a1 together with the resistant plasmid. Neurons were stained for SULT4A1 (red) to 
A
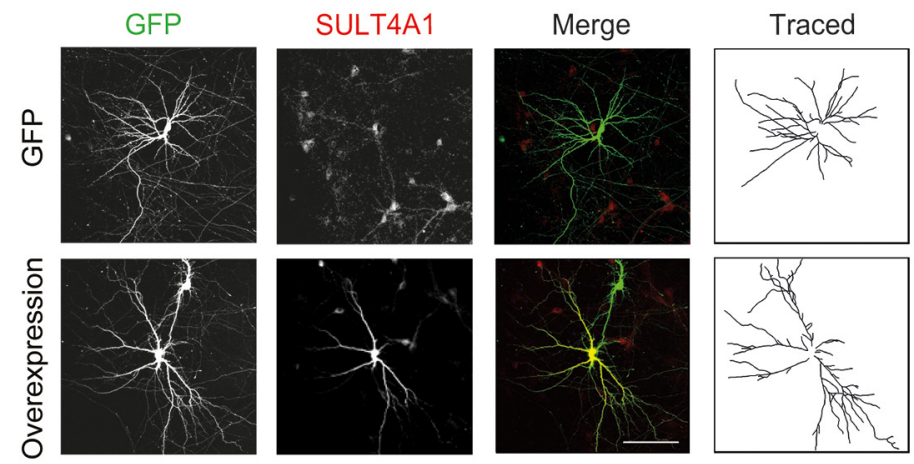

B
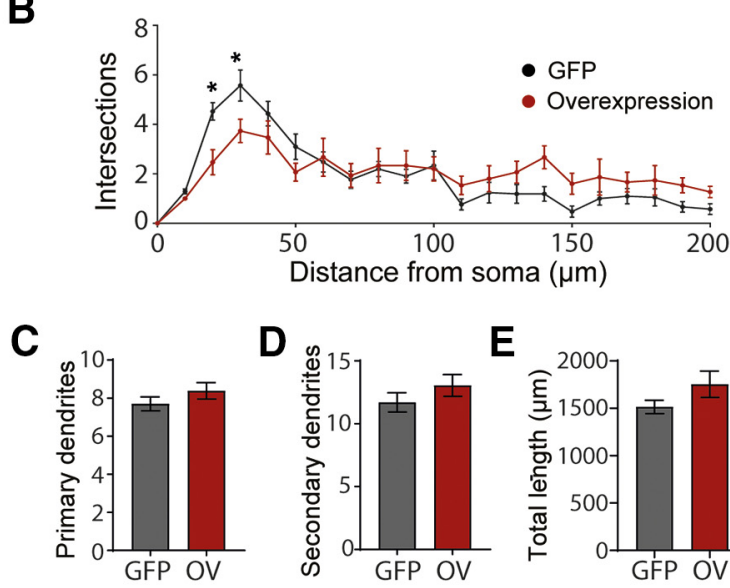

E

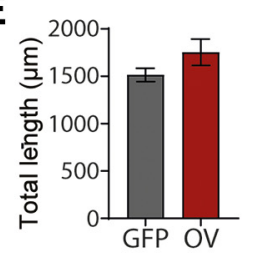

F

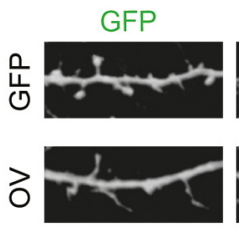

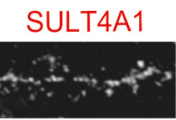

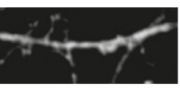

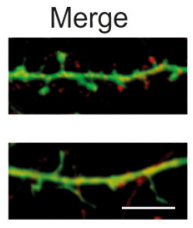

G

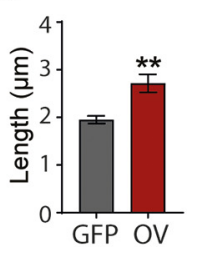

H

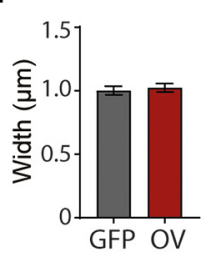

I

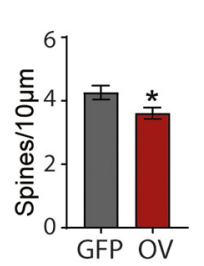

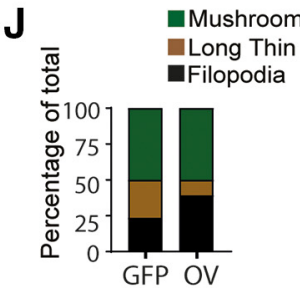

Figure 3. Sult4a1 overexpression (OV) impairs dendritic arborization and spines. $A$, Representative immunofluorescence images and relative traces of rat cortical neurons transfected with pLVTHM alone (GFP) or together with pFlag-SULT4A1 (overexpression). Neurons were stained for SULT4A1 (red) to assess protein (over)expression. Scale bar, $100 \mu \mathrm{m}$. $\boldsymbol{B}$, Results of Sholl analysis and quantification of the number of intersections, plotted against the distance from the soma (GFP, $n=21$ individual neurons; overexpression, $n=15$ individual neurons; two-way ANOVA, $p<0.0001$; Sidak's post hoc test: $\left.10 \mu \mathrm{m},{ }^{*} p=0.0126 ; 20 \mu \mathrm{m},{ }^{*} p=0.0444\right)$. C, D, Quantification of the number of primary ( $\boldsymbol{C}$ ) and secondary (D) dendrites (GFP, $n=20$ individual neurons; overexpression, $n=21$ individual neurons; for $C$ : Mann-Whitney test, $p=0.3511$; for $\boldsymbol{D}$ : unpaired $t$ test, $p=0.2496$ ). $\boldsymbol{E}$, Quantification of total dendrites length (GFP $n=20$ individual neurons, Overexpression, $n=21$ individual neurons; unpaired $t$ test, $p=0.2387$ ). $\boldsymbol{F}$, Representative immunofluorescence images showing dendritic spines of rat cortical neurons transfected with pLVTHM alone (GFP) or together with pFlag-SULT4A1 (OV). Neurons were stained for SULT4A1 (red) to assess protein (over)expression. Scale bar, $10 \mu \mathrm{m}$. $G-I$, Quantification of dendritic spines length $(\mathbf{G})$, width $(\boldsymbol{H})$, and number of spines per $10 \mu \mathrm{m}$ of dendrite $\left(\boldsymbol{I} ; \mathrm{GFP}, n=15\right.$ individual dendrites; $0 \mathrm{~V}, n=23$ individual dendrites; unpaired $t$ test, $\left.\mathbf{G},{ }^{* *} p=0.0126 ; \boldsymbol{I},{ }^{*} p=0.0303\right)$. $\boldsymbol{J}$, Quantification of filopodia, long thin and mushroom spines distribution. Data represent the mean \pm SEM.

antibody to identify Sult4a1-overexpressing neurons (Fig. 3A). Sult4a1 overexpression led to an overall redistribution of the branching points along the dendritic tree compared with wildtype neurons: neurons overexpressing Sult4a1 presented fewer branching points close to the soma $(<50 \mu \mathrm{m}$ from soma) and an increased number of distal branching points $(>100 \mu \mathrm{m}$ from soma; condition by distance interaction, $p=0.0005$; Fig. $3 B$ ), suggesting a reorganization of neuronal arborization. However, the

assess protein expression. Scale bar, $10 \mu \mathrm{m} . \mathbf{G}-\mathbf{I}$, Quantification of dendritic spines length $(\boldsymbol{G})$, width $(\boldsymbol{H})$, and number of spines per $10 \mu \mathrm{m}$ of dendrite $(\boldsymbol{l} ;$ shCtrl, $n=10$ individual dendrites; shSult4a1, $n=10$ individual dendrites; Sult4a1r, $n=14$ individual dendrites; KruskalWallis test: $p=0.6092$ for $\boldsymbol{G}, p=0.8472$ for $\boldsymbol{H}, p=0.0081$ for $\boldsymbol{l}$; $\boldsymbol{l}$ : Dunn's post hoc test: shCtrl vs shSult4a1, $p=0.0105$; shSult4a1 vs Sult4a1r, $p=0.0417$; ns, not significant). $J$, Quantification of filopodia, long thin and mushroom spines distribution. $\boldsymbol{K}$, Representative immunohistochemistry images from brain slices obtained from 30-d-old in utero electroporated mice, showing cortical neurons expressing shCtrl or shSult4a1 and their relative traces. Scale bar, $100 \mu \mathrm{m}$. L, Results of Sholl analysis and quantification of the number of intersections, plotted against the distance from the soma (shCtrl, $n=10$ individual neurons; shSult4a1, $n=13$ individual neurons; two-way ANOVA, $p=0.0629$; Sidak's post hoc test, $\left.60 \mu \mathrm{m}:{ }^{*} p=0.0162 ; 70 \mu \mathrm{m}:{ }^{* * * *} p<0.0001\right)$. M, Quantification of total dendrites length (shCtrl, $n=10$ individual neurons; shSult4a1, $n=13$ individual neurons; unpaired $t$ test, ${ }^{* * *} p=0.0002$ ). $\boldsymbol{N}$, Representative immunofluorescence images showing dendritic spines of shCtrl- or shSult4a1-expressing cortical neurons from 30-d-old in utero electroporated mice. Scale bar, $10 \mu \mathrm{m} . \mathbf{0}-\mathbf{Q}$, Quantification of dendritic spines length $(\mathbf{0})$, width $(\boldsymbol{P})$, and number of spines per $10 \mu \mathrm{m}$ of dendrite $(\boldsymbol{Q}$; shCtrl, $n=12$ individual dendrites; shSult4a1, $n=9$ individual dendrites; unpaired $t$ test $p=0.0490)$. $R$, Quantification of thin, stubby, and mushroom spines distribution. Data represent the mean \pm SEM. shSult4a1 vs Sult4a1r, ${ }^{* *} p=$ 0.0079 . number of primary dendrites (GFP, $7.7 \pm 0.36$; overexpression, $8.38 \pm 0.43$ ), secondary dendrites (GFP, $11.7 \pm 0.76$; overexpression, 13.05 \pm 0.86 ), and the total dendrites length (GFP, 1513.0 \pm 70.64 ; overexpression, $1753.32 \pm 139.04)$ were equal in the two conditions (Fig. 3C-E). Interestingly, cortical neurons overexpressing Sult4al displayed a significant decrease in dendritic spine density (Fig. 3I; spine number: GFP, $4.26 \pm 0.22$; overexpression, $3.61 \pm 0.18$ ) and correlated with considerably longer spine morphology, compared with wild-type neurons, suggesting an immature phenotype (Fig. $3 G$ : spine length: GFP, $1.95 \pm 0.08$ $\mu \mathrm{m}$; overexpression, $2.71 \pm 0.19 \mu \mathrm{m}$; Fig. $3 H$ : spine width: GFP, $1 \pm 0.03 \mu \mathrm{m}$; overexpression, $1.02 \pm 0.03 \mu \mathrm{m}$; Fig. $3 F-I)$. Indeed, we found an increase in spines with filopodia-like morphology in neurons overexpressing Sult4a1 (Fig. 3J).

These data demonstrate an important role for SULT4A1 in the regulation of both dendritic complexity and dendritic spine morphology.

Functional modifications induced by SULT4A1 knockdown Considering that SULT4A1 knockdown affected dendritic arborization and spine density, the effect of SULT4A1 silencing on the expression of relevant synaptic proteins was further investigated.

First, a lentiviral plasmid expressing shSult4al was validated as effective in reducing the levels of endogenous SULT4A1 protein in cortical neurons (Fig. $4 A$; Ni 1; shCtrl, $0.94 \pm 0.02$; shSult4a1, $0.08 \pm 0.02$ ). Immunoblotting analysis of total lysates showed a significant decrease in NMDA receptor 
A
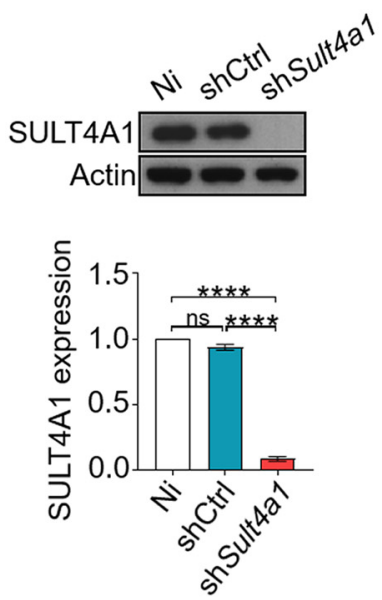

B

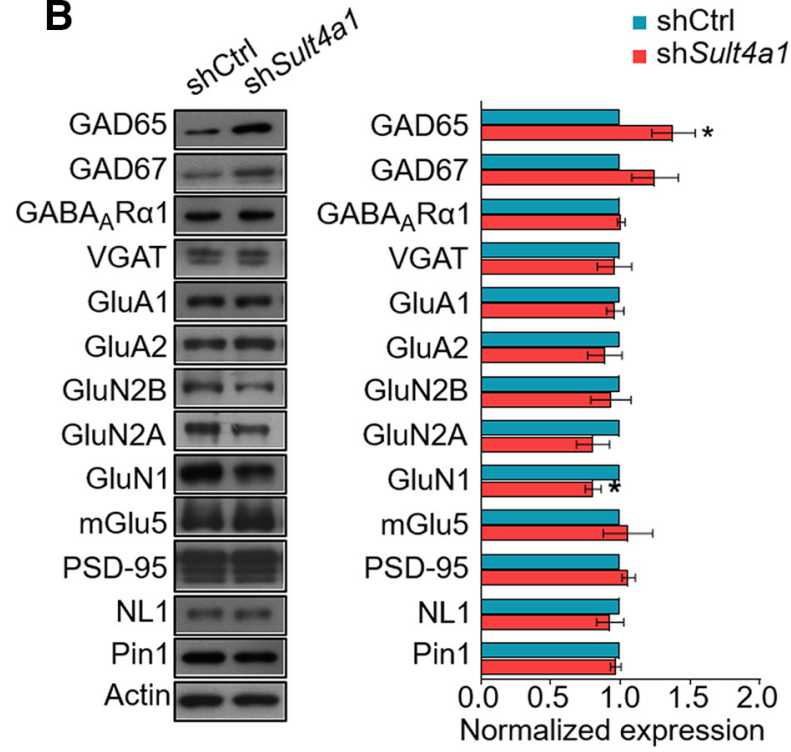

C

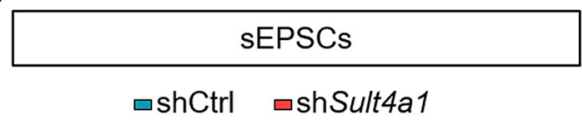

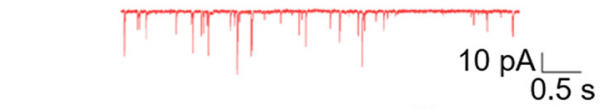
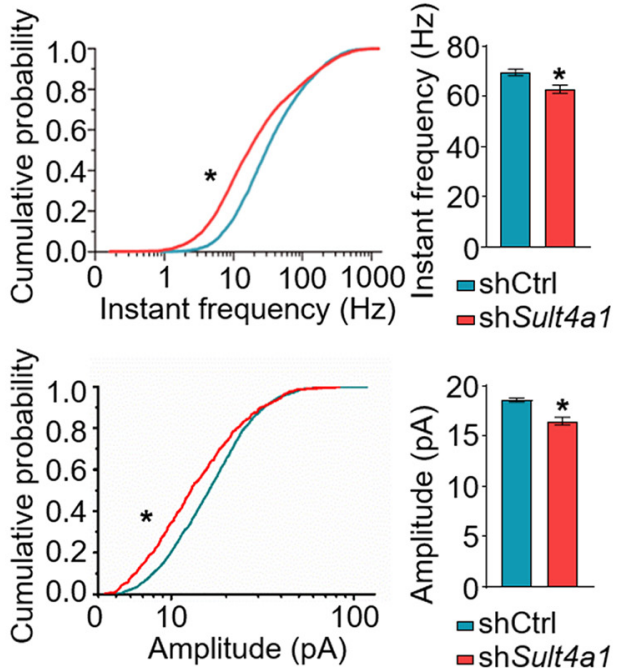

D
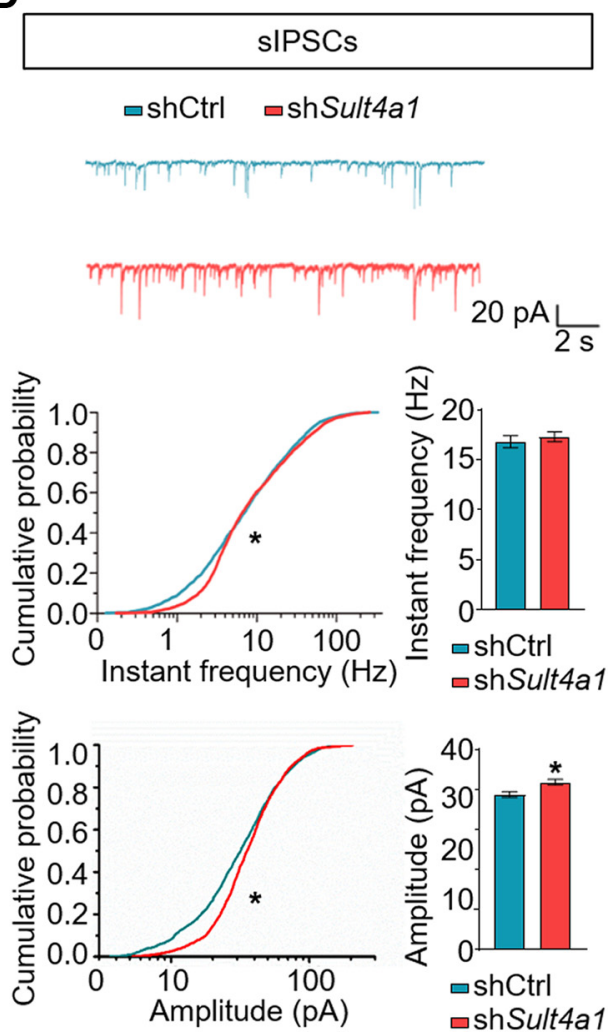

Figure 4. Sult4a1 knockdown alters synaptic transmission. $\boldsymbol{A}$, Representative immunoblots of total protein lysates derived from DIV14 rat cortical neurons transduced with a lentivirus expressing shCtrl or shSult4a1. Both conditions were compared with not infected condition (Ni) so as to verify whether the infection itself could cause any alteration of Sult4a1 protein expression. Sult4a1 protein levels were normalized against the level of the not infected neurons ( $\mathrm{Ni}, n=7$ independent cultures; shCtrl, $n=4$ independent cultures; shSult4a1, $n=7$ independent cultures; one-sample $t$ test: Ni vs shSult4a1, ${ }^{* * *} p<0.0001$; shCtrl vs shSult4a1, ${ }^{* * *} p<0.0001$; ns, not significant). $\boldsymbol{B}$, Representative Western blots of total lysates from shCtrl- or shSult4a1transduced cortical neurons. Protein levels were normalized against the level of the shCtrl-transduced neurons (shCtrl, $n \geq 3$ independent cultures; shSult4a1, $n \geq 3$ independent cultures; onesample $t$ test: GAD65, ${ }^{*} p=0.0396$; GluN1, ${ }^{*} p=0.0186$ ). C, Top panels, Representative sEPSCs recorded from shCtrl-transfected (blue) or shSult4a1-transfected (red) neurons. Middle panels, left, Plot of cumulative probability of sEPSC frequencies ( $\mathrm{shCtrl,} n=5$ individual neurons; shSult4a1, $n=5$ individual neurons; Kolmogorov-Smirnov test, $p=4 \times 10^{-18}$ ); right, mean instantaneous frequencies plot (shCtrl, $n=5$ individual neurons; shSult4a1, $n=5$ individual neurons; Mann-Whitney test, ${ }^{*} p<0.05$; data are shown as the mean \pm SEM). Bottom panels, left, Plot of cumulative probability of sEPSC amplitudes (Kolmogorov-Smirnov test, $p=10^{-11}$ ); right, plot of mean sEPSC amplitudes (shCtrl, $n=5$ individual neurons; shSult4a1, $n=5$ individual neurons; Mann-Whitney test, $p=10^{-12}$; data are shown as the mean \pm SEM). $\boldsymbol{D}$, Top panels, Representative sIPSCs recorded from shCtrl-transfected (blue) or shSult4a1-transfected (red) neurons. Middle panels, Left, Plot of cumulative probability of sIPSC frequencies (shCtrl, $n=5$ individual neurons; shSult4a1, $n=6$ individual neurons; Kolmogorov-Smirnov test, $p=5 \times 10^{-8}$ ); right, plot of mean instantaneous frequencies (shCtrl, $n=5$ individual neurons; shSult4a1, $n=6$ individual neurons; Mann-Whitney test; data are shown as the mean \pm SEM). Bottom panels, Left, Plot of cumulative probability of sIPSC amplitudes (Kolmogorov-Smirnov test, $p=10^{-12}$ ); right, plot of mean sIPSC amplitudes (shCtrl, $n=5$ individual neurons; shSult4a1, $n=5$ individual neurons; Mann-Whitney test, $p=10^{-8}$; data are shown as the mean \pm SEM). 
A
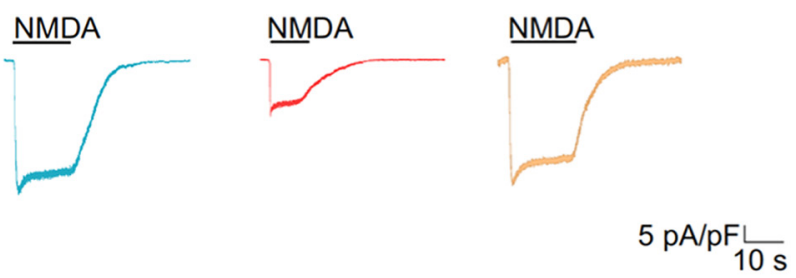

B
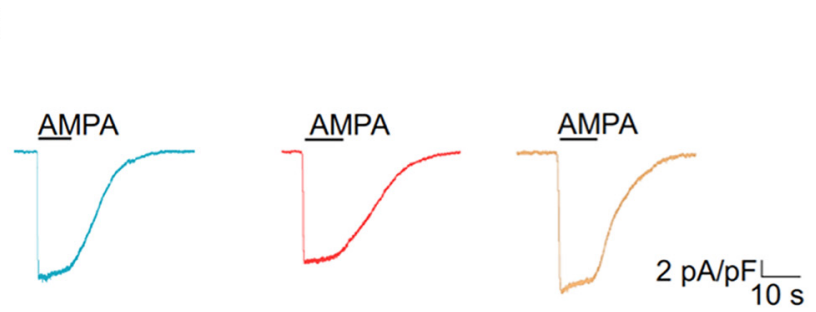
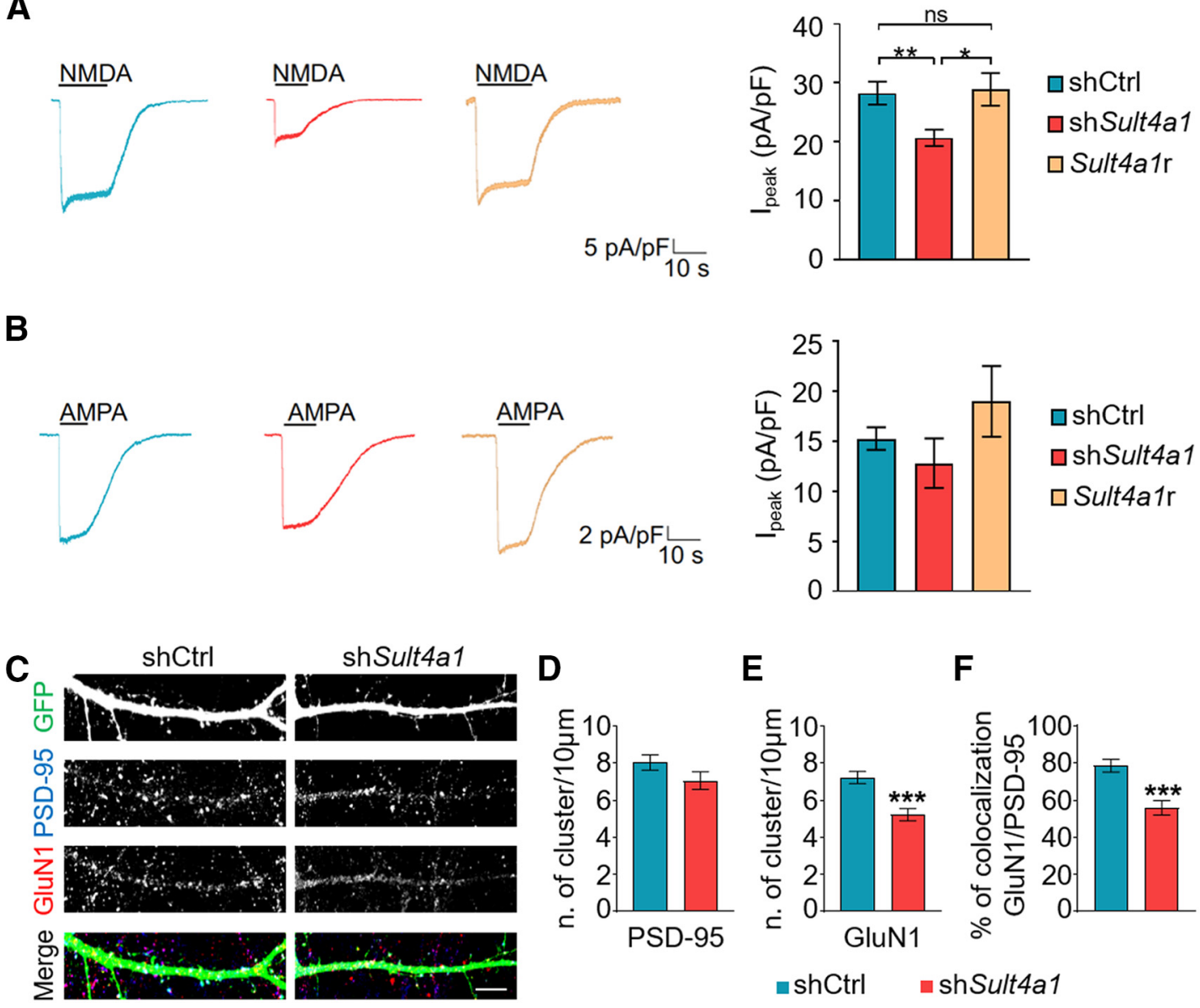

shSult4a1

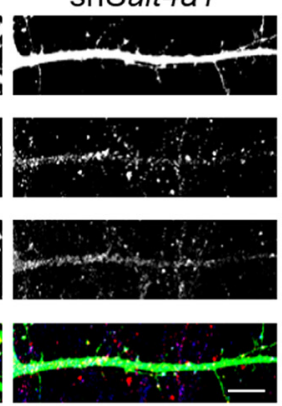

D

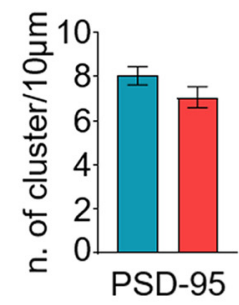

E

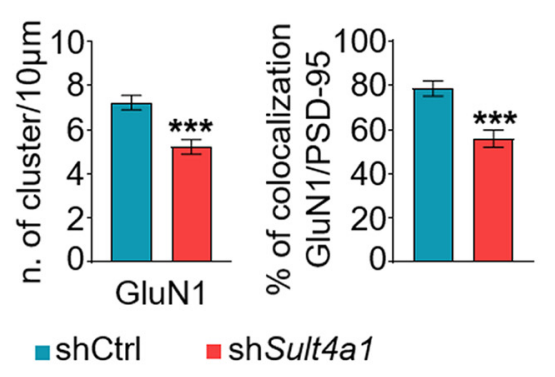

G

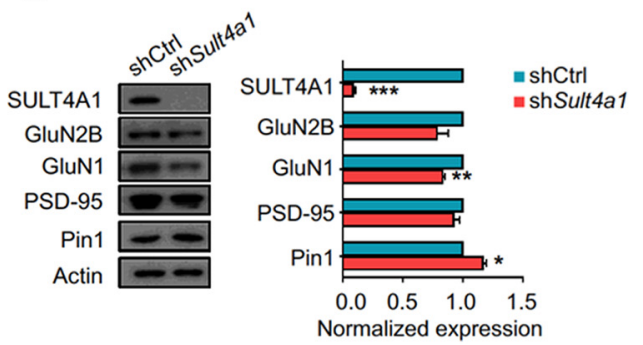

H

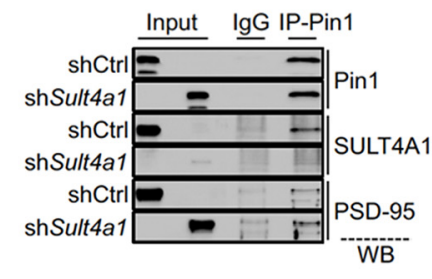

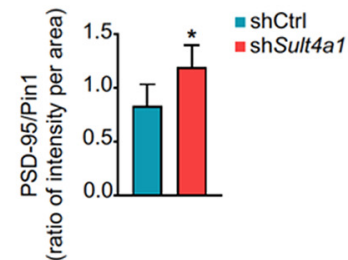

Figure 5. SULT4A1 interaction with Pin 1 and its role in excitatory synapses. $A$, Left, Representative $I_{\text {NMDA }}$ traces elicited by the perfusion of $100 \mu$ MMDA from neurons transfected with shCtrl (blue), shSult4a1 (red), or shSult4a1 together with the resistant plasmid Sult4a1r (orange). Right, Analysis of the mean peak I IMDA current densities in neurons transfected with shCtrl, shSult4a1, or shSult4a1 together with the resistant plasmid Sult4a1r (shCtrl, $n=30$ individual neurons; shSult4a1, $n=34$ individual neurons; Sult4a1r, $n=10$; one-way AN0VA, $p=0.0028$; Tukey's post hoc test: shCtrl vs shSult4a1, $p=0.0048$; shSult4a1 vs Sult4a1r, $p=0.0423$; ns, not significant). shCtrl vs shSult4a1, ${ }^{* *} p=0.0048$; shSult4a1 vs Sult4a1r, ${ }^{*} p=0.0423$. $\boldsymbol{B}$, Left, Representative $I_{\text {AMPA }}$ traces elicited by the perfusion of $100 \mu \mathrm{M}$ AMPA from neurons transfected with shCtrl (blue), shSult4a1 (red), or shSult4a1 together with the resistant plasmid Sult4a1r (orange). Right, Analysis of mean peak $I_{\text {AMPA }}$ current densities in neurons transfected with shCtrl, shSult4a1, or shSult4a1 together with the resistant plasmid Sult4a1r (shCtrl, $n=11$ individual neurons; shSult4a1, $n=8$ individual neurons; Sult4a1r, $n=7$ individual neurons; one-way ANOVA, $p=0.2133$ ). $C$, Representative immunofluorescence images showing dendritic spines of rat cortical neurons transfected with shCtrl or shSult4a1. Neurons were stained for PSD-95 (blue) and GluN1 (red). Scale bar, $10 \mu \mathrm{m}$. D, E, Quantification of the number of PSD-95 (D) and GluN1 (E) clusters per $10 \mu \mathrm{m}$ of dendrite (shCtrl, $n=11$ individual dendrites; shSult4a1, $n=11$ individual dendrites; unpaired $t$ test, $\boldsymbol{E}, p=0.0003$ ). $\boldsymbol{F}$, Quantification of GluN1 and PSD-95 clusters colocalization (shCtrl, $n=11$ individual dendrites; shSult4a1, $n=11$, individual dendrites; unpaired $t$ test, ${ }^{* * *} p=0.0004$ ). G, Representative Western blots of synaptosomal fractions obtained from cortical neurons transduced with shCtrl or shSult4a1. Protein levels were normalized against the levels of the shCtrl-transduced neurons (shCtrl, $n \geq 3$ independent cultures; shSult4a1, $n \geq 3$ independent cultures; one-sample $t$ test, SULT4A1, $p<0.0001$; GluN1, $p=0.0032 ;$ Pin1, $p=0.0206$ ). $\boldsymbol{H}$, Left, Representative images of immunoprecipitation (IP) assay performed on synaptosomal fractions derived from neurons transduced with shCtrl or shSult4a1. Proteins were precipitated using mouse anti-Pin 1 or mouse lgG antibodies, and nitrocellulose membranes were probed with anti-Pin1, anti-SULT4A1, and anti-PSD-95 antibodies (WB). Right, Histogram showing the ratio between PSD-95 and Pin1 signals (PSD-95/Pin1; shCtrl, $n=4$ independent experiments; shSult4a1, $n=4$ independent experiments; unpaired $t$ test, $p=0.0464)$. Data represent the mean \pm SEM. ${ }^{*} p=0.0464$.

subunit GluN1 expression (shCtrl, 1; shSult4a1, $0.81 \pm 0.06$ ) and, simultaneously, a considerable increase of GAD65 protein level (shCtrl, 1; shSult4a1, 1.38 \pm 0.16 ) in shSult4a1-infected neurons (Fig. 4B).
To explore whether SULT4A1 silencing functionally affected glutamatergic and GABAergic activity, spontaneous postsynaptic currents (sPSCs) were recorded from shCtrl or shSult4a1 transfected neurons. SULT4A1 knock-down neurons displayed a 
significant reduction of sEPSC frequency and amplitude, reflected by the leftward shift of the cumulative probability curves and by the reduction of the median value (instantaneous frequency: shCtrl: median, $30.0 \mathrm{~Hz}$; mean \pm SEM, $69.6 \pm 1.3 \mathrm{~Hz}$; shSult4a1: median, $16.9 \mathrm{~Hz}$; mean $\pm \mathrm{SEM}, 62.9 \pm 1.6 \mathrm{~Hz}$; amplitude: shCtrl: median, $16.1 \mathrm{pA}$; mean \pm SEM, $18.6 \pm 0.2 \mathrm{pA}$; shSult4a1: median, $12.9 \mathrm{pA}$; mean \pm SEM, $16.5 \pm 0.4 \mathrm{pA}$; Fig. $4 C)$. Moreover, an increase of the frequency of sIPSCs was observed as a reduction of low-frequency events (range, 0.5-5 $\mathrm{Hz}$ ), reflected by the rightward shift of the lower part of the cumulative probability curve, whereas the median value displayed a nonsignificant trend toward increased frequency (shCtrl: median, $7.0 \mathrm{~Hz}$; mean \pm SEM, $16.8 \pm 0.6 \mathrm{~Hz}$; shSult4a1: median, $5.5 \mathrm{~Hz}$; mean \pm SEM, $17.3 \pm 0.5 \mathrm{~Hz}$; Fig. $4 D$ ). The amplitude of sIPSCs was instead significantly increased, reflected by the rightward shift of the cumulative probability curve and by the increase of the median value sIPSCs (shCtrl: median, $31.8 \mathrm{pA}$; mean \pm SEM, $38.7 \pm 0.7 \mathrm{pA}$; shSult4a1: median, $35.5 \mathrm{pA}$; mean \pm SEM, $41.8 \pm 0.7 \mathrm{pA})$. Consistently with data on expression levels, the results of the analysis of sPSCs suggest that SULT4A1 knockdown induces a decrease of glutamatergic synaptic activity and an increase of GABAergic synaptic activity.

\section{SULT4A1 modulates NMDAR activity preventing Pin1- PSD-95 interaction}

Our biochemical data indicate that the knockdown of SULT4A1 in neurons causes a significant decrease of GluN1. Therefore, total $I_{\mathrm{NMDA}}$ values in neurons transfected with shSult4a1 were assessed. A specific reduction of $I_{\mathrm{NMDA}}$ was observed, quantified as the peak current density. This reduction was prevented by cotransfecting the shSult4a1 with the Sult4a1r (shCtrl, 28.3 \pm 1.9 pA/pF; shSult4a1, $20.7 \pm 1.4 \mathrm{pA} / \mathrm{pF}$; Sult4alr, $28.9 \pm 2.7 \mathrm{pA} / \mathrm{pF}$; Fig. 5A). Moreover, we measured total $I_{\mathrm{AMPA}}$. Our results showed a slight but not significant reduction in neurons transfected with shSult4al (shCtrl, $15.3 \pm 1.1 \mathrm{pA} / \mathrm{pF}$; shSult4a1, $12.8 \pm 2.4 \mathrm{pA} / \mathrm{pF}$; Sult4a1r, $18.9 \pm 3.5 \mathrm{pA} / \mathrm{pF}$; Fig. $5 B)$, suggesting that SULT4A1 knockdown caused a specific NMDAR impairment in synapses.

To elucidate whether the decreased $I_{\mathrm{NMDA}}$ was associated with a reduced recruitment of NMDAR clusters at synapses, immunocytochemical experiments were performed on cortical neurons colabeled for PSD-95 and GluN1 (Fig. 5C). We found a significant reduction in the number of GluN1 clusters per $10 \mu \mathrm{m}$ of dendrite (shCtrl, 7.23 \pm 0.33 ; shSult4a1, 5.22 \pm 0.33 ) and a reduction of GluN1/PSD-95 colocalization (percentage of colocalization: shCtrl, $78.7 \pm 3.41$; shSult4a1, 55.88 \pm 3.90$)$ in Sult4a1 knock-down neurons (Fig. $5 E, F$ ). No difference in the number of PSD-95 clusters was detected (shCtrl, 8.04 \pm 0.41 ; shSult4a1, $7.06 \pm 0.47$; Fig. $5 D)$.

Immunoblot analysis confirmed lower levels of GluN1 (shCtrl, 1; shSult4a1, $0.83 \pm 0.02$ ) and, albeit not statistically significant, a trend toward a reduction of GluN2B $(p=0.1084$; shCtrl, 1 ; shSult4a1, $0.7852 \pm 0.10$ ) in synaptosomal preparations derived from Sult4a1-deficient neurons (Fig. 5G).

Peptidyl-prolyl cis-trans isomerase Pin 1 is highly expressed at excitatory synapses, where it exerts a negative action on synaptic transmission by interfering with the PSD-95/GluN2B complex formation (Antonelli et al., 2016). Since it has been demonstrated that SULT4A1 is able to specifically interact with Pin1 with the phosphoserine/threonine-proline motifs in its $\mathrm{N}$ terminus (Mitchell and Minchin, 2009), the effects of SULT4A1 knockdown on synaptic expression of Pin 1 was analyzed. A significant increase in Pin 1 synaptic levels was evidenced by immunoblot analysis of synaptosomal preparations derived from SULT4A1deficient neurons (shCtrl, 1; shSult4a1, $1.17 \pm 0.02$; Fig. $5 G$ ).

To test whether SULT4A1 directly affects the Pin1-PSD-95 interaction at synaptic levels, endogenous Pin1 was immunoprecipitated from synaptosomal preparations using an anti-Pin1 antibody, and the coprecipitated PSD-95 was visualized using an anti-PSD-95 antibody (Fig. 5H). With SULT4A1 silencing, the amount of PSD-95 coprecipitated by Pin1 was increased by $40 \%$ compared with SULT4A1-expressing neurons (shCtrl, $0.83 \pm$ 0.10 ; shSult4a1, $1.19 \pm 0.10$ ). Thus, these results suggest that SULT4A1 is able to recruit Pin1 and, preventing its interaction with PSD-95, facilitates the formation of PSD-95/NMDAR complex. In conclusion, our data suggest that the reduction of NMDAR-mediated currents in SULT4A1 knocked-down neurons is mediated by the increased interaction of Pin 1 with PSD95 in excitatory synapses.

\section{Reversal of morphologic and functional deficits via Pin1 inhibition}

As the synaptic level of Pin1 was found to be significantly increased following SULT4A1 knockdown, the role of Pin 1 catalytic activity was assessed as a potential mechanism underlying altered glutamatergic transmission. The selective Pin1 inhibitor $\mathrm{PiB}$ was used to inhibit Pin1 activity in SULT4A1 knock-down neurons.

Cortical neurons transfected with shSult4a1 or shCtrl at DIV7 were acutely treated with $2.5 \mu \mathrm{M} \mathrm{PiB}$ or vehicle (DMSO) at DIV12 for $48 \mathrm{~h}$. Acute treatment with $\mathrm{PiB}$ resulted in an increase in peak current density in SULT4A1 knocked-down neurons, while no significant effect was observed in neurons transfected with shCtrl. (shCtrl vehicle, $33.1 \pm 3.3 \mathrm{pA} / \mathrm{pF}$; shCtrl PiB, $29.8 \pm 3.5 \mathrm{pA} / \mathrm{pF}$; shSult4a1 vehicle, $22.2 \pm 1.2 \mathrm{pA} / \mathrm{pF}$; shSult4a1 $\mathrm{PiB}, 32 \pm 2.6 \mathrm{pA} / \mathrm{pF}$; Fig. $6 A$ ). A similar result was observed by acute treatment with $4 \mu \mathrm{M}$ DTM, an additional Pin 1 chemical inhibitor (Tatara et al., 2010; shCtrl vehicle, $29.0 \pm 4.7 \mathrm{pA} / \mathrm{pF}$; shCtrl DTM, $30.8 \pm 7.1 \mathrm{pA} / \mathrm{pF}$; shSult4a1 vehicle, $22.2 \pm 3.5 \mathrm{pA} /$ pF; shSult4a1 DTM, $42.7 \pm 7.7$ pA/pF; Fig. 6B). Thus, Pin1 inhibition can revert NMDA currents alteration induced by SULT4A1 loss of function.

Additionally, the effect of Pin 1 inhibition on dendritic spine density was investigated. Acute treatment with $\mathrm{PiB}$ was able to restore the number of dendritic spines in SULT4A1 knockeddown neurons to levels comparable to those of control neurons (spine number: shCtrl vehicle, $5.67 \pm 0.30$; shCtrl $\mathrm{PiB}, 6.12 \pm$ 0.42; shSult4a1 vehicle, $4.33 \pm 0.20$; shSult4a1 $\mathrm{PiB}, 5.82 \pm 0.30$; Fig. $6 C$ ). The spine number was also rescued by acute treatment with the second Pin1 inhibitor, DTM (spine number: shCtrl vehicle, $6.78 \pm 0.25$; shCtrl DTM, 7.6 \pm 0.5 ; shSult4a1 vehicle, $4.8 \pm 0.37$; shSult4a1 DTM, $7.8 \pm 0.7$; Fig. $6 D$ ).

To revert dendritic arborization deficits, shSult4a1- and shCtrl-transfected neurons were treated every other day with 1 $\mu \mathrm{M} \mathrm{PiB}$ or $4 \mu \mathrm{M}$ DTM or vehicle, starting at DIV7. However, chronic treatment with neither PiB nor DTM had any significant effect on neuronal branching ( $\mathrm{PiB}$ treatment: shCtrl vehicle vs PiB: treatment factor, $p=0.4881$; treatment by distance interaction, $p=0.9999$; shSult4a1 vehicle vs $\mathrm{PiB}$ : treatment factor, $p=0.3740$; treatment by distance interaction, $p=0.9671$; DTM treatment: shCtrl vehicle vs DTM: treatment factor, $p=0.0743$; treatment by distance interaction, $p=0.9021$; shSult4a1 vehicle vs DTM: treatment factor, $p=0.6666$; treatment by distance interaction, $p=0.0886$; Fig. $7 A-F)$. 
A
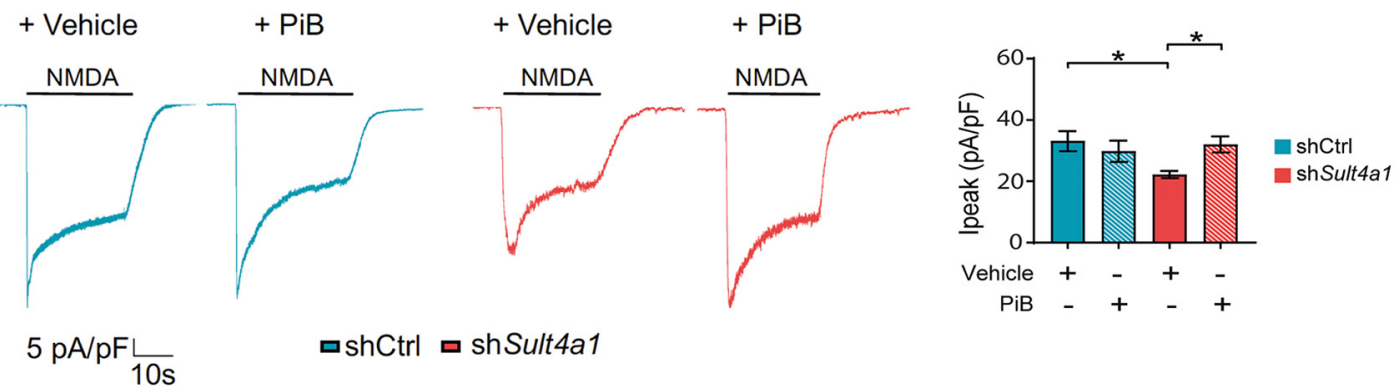

B

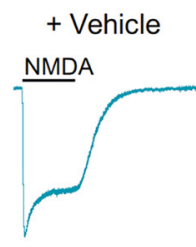

+ DTM

+ Vehicle

+ DTM

NMDA $\underline{\text { NMDA }}$

$5 \mathrm{pA} / \mathrm{pF} \frac{\mathrm{L}}{10 \mathrm{~s}}$

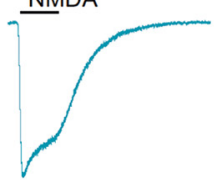

NMDA

NMDA
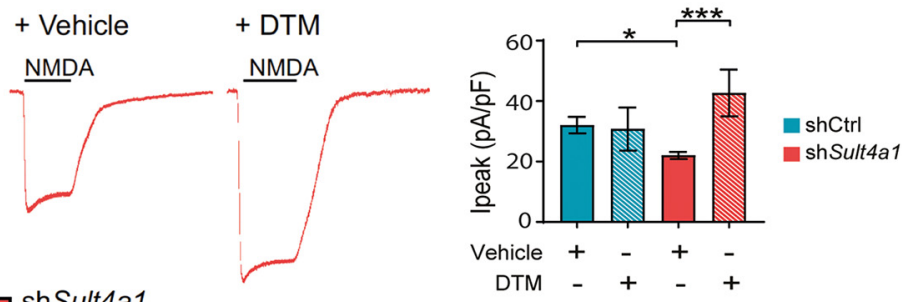

C

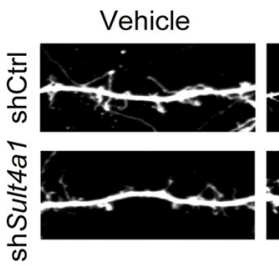

$\mathrm{PiB}$
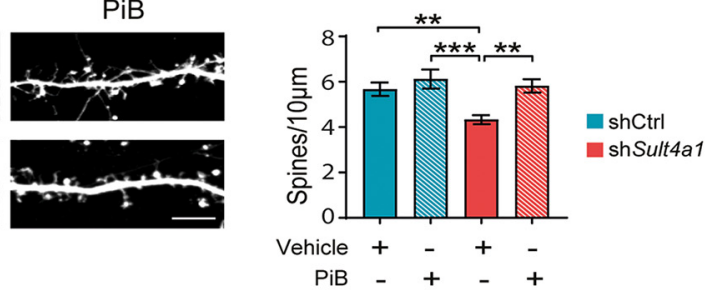

E

D
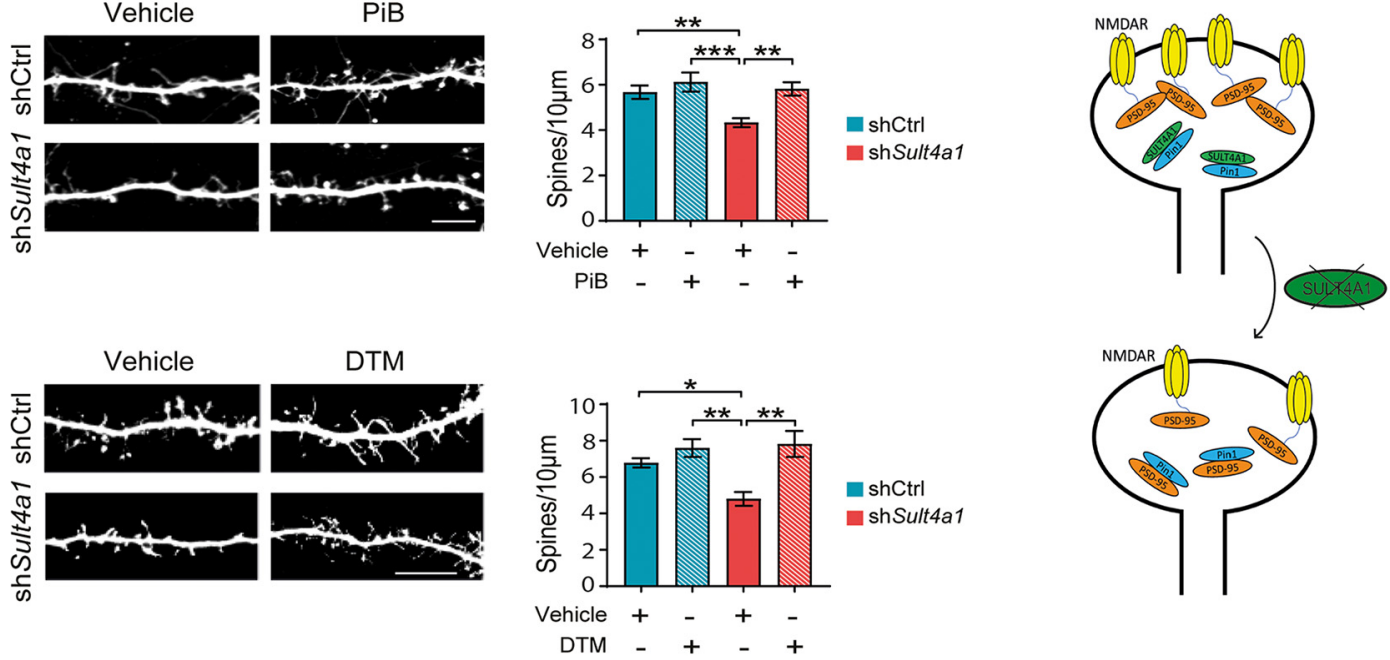

Figure 6. Rescue of shSult4a1-dependent phenotypes via Pin1 pharmacological inhibition. $\boldsymbol{A}$, Left, Representative $I_{\text {NMDA }}$ traces from shCtrl-transfected (blue) and shSult4a1-transfected (red) neurons, treated for $48 \mathrm{~h}$ with $2.5 \mu \mathrm{MPiB}$ or vehicle. Right, Analysis of mean peak $I_{\mathrm{NMDA}}$ current densities (shCtrl + vehicle, $n=12$ individual neurons; shCtrl $+\mathrm{PiB}, n=16$ individual neurons; shSult4a1 + vehicle, $n=6$ individual neurons; shSult4a1 + PiB, $n=16$ individual neurons; one-way ANOVA, $p=0.0082$; Tukey's post hoc test: shCtrl vehicle vs shSult4a1 vehicle, $p=0.0146$; shSult4a1 vehicle vs shSult4a1 PiB, $p=0.0186$ ). shCtrl vehicle vs shSult4a1 vehicle, ${ }^{*} p=0.0146$; shSult4a1 vehicle vs shSult4a1 PiB, ${ }^{*} p=0.0186$. $B$, Left, Representative $I_{\text {NMDA }}$ traces from shCtrl-transfected (blue) and shSult4a1-transfected (red) neurons, treated for $48 \mathrm{~h}$ with $4 \mu \mathrm{m}$ DTM or vehicle. Right, Analysis of mean peak $I_{\text {MMDA }}$ current densities (shCtrl + vehicle, $n=16$ individual neurons; shCtrl + DTM, $n=7$ individual neurons; shSult4a1 + vehicle, $n=21$ individual neurons; shSult4a1 + DTM, $n=10$ individual neurons; one-way AN0VA, $p=0.0008$; Tukey's post hoc test: shCtrl + vehicle vs shSult4a1 + vehicle, $p=0.0455$; shSult4a1 + vehicle vs shSult4a1 + DTM, $p=0.0006)$. shCtrl vehicle vs shSult4a1 vehicle, ${ }^{*} p=0.0455$; shSult4a1 vehicle vsshSult4a 1 DTM, ${ }^{* * *} p=0.0006$. C, Left, Representative immunofluorescence images showing dendritic spines of rat cortical neurons transfected with shCtrl or shSult4a1 and treated for $48 \mathrm{~h}$ with $2.5 \mu \mathrm{m} \mathrm{PiB}$, a pharmacological inhibitor of Pin1 catalytic activity, or vehicle (DMSO). Scale bar, $10 \mu \mathrm{m}$. Right, Quantification of the number of dendritic spines per $10 \mu \mathrm{m}$ of dendrite (shCtrl + vehicle, $n=19$ individual dendrites; shCtrl + PiB, $n=16$ individual dendrites; shSult4a1 + vehicle, $n=19$ individual dendrites; shSult4a1 + PiB, $n=17$ individual dendrites; one-way ANOVA, $p=0.0003$; Tukey's post hoc test: shCtrl + vehicle vs shSult4a1 + vehicle, $p=0.0096$; shCtrl + PiB vs shSult4a1 vehicle, $p=0.0005$; shSult4a1 + vehicle vs shSult4a1 + PiB, $p=0.0044)$. shCtrl vehicle vs shSult4a1 vehicle, ${ }^{* *} p=0.0096$; shCtrl PiB vs shSult4a vehicle, ${ }^{* * *} p=0.0005$; shSult4avehicle vs shSult4a1 PiB ${ }^{* *} p=0.0044$. D, Left, Representative immunofluorescence images showing dendritic spines of rat cortical neurons transfected with shCtrl or shSult4a1 and treated at DIV12 for $48 \mathrm{~h}$ with $4 \mu \mathrm{m}$ DTM, a pharmacological inhibitor of Pin1 catalytic activity, or vehicle (DMSO). Scale bar, $10 \mu \mathrm{m}$. Right, Quantification of the number of dendritic spines per $10 \mu \mathrm{m}$ of dendrite (shCtrl + vehicle, $n=10$ individual dendrites; shCtrl + DTM, $n=10$ individual dendrites; shSult4a1 + vehicle, $n=11$ individual dendrites; shSult4a $1+$ DTM, $n=10$ individual dendrites; Kruskal-Wallis test, $p=0.0006$; Dunn's post hoc test: shCtrl + vehicle vs shSult4a1 + vehicle, $p=0.0301$; shCtrl + DTM vs shSult4a1 vehicle, $p=0.0012$; shSult4a1 + vehicle vs shSult4a1 + DTM, $p=0.0005)$. shCtrl vehicle vs shSult4a1 vehicle, ${ }^{*} p=0.0301$; shCtrl DTM vs shSult4a1 vehicle, ${ }^{* *} p=0.0012$; shSult4a1 vehicle vs shSult4a1 DTM, ${ }^{* *} p=0.0005$ ). $\boldsymbol{E}$, Cartoon showing the hypothesized mechanism. SULT4A1 interacts with Pin1 promoting the formation of the PSD-95/NMDAR complex in excitatory synapses. In the absence of SULT4A1, Pin1 is free to interact with PSD-95, reducing NMDAR expression in synapses. Data represent the mean \pm SEM. 
A

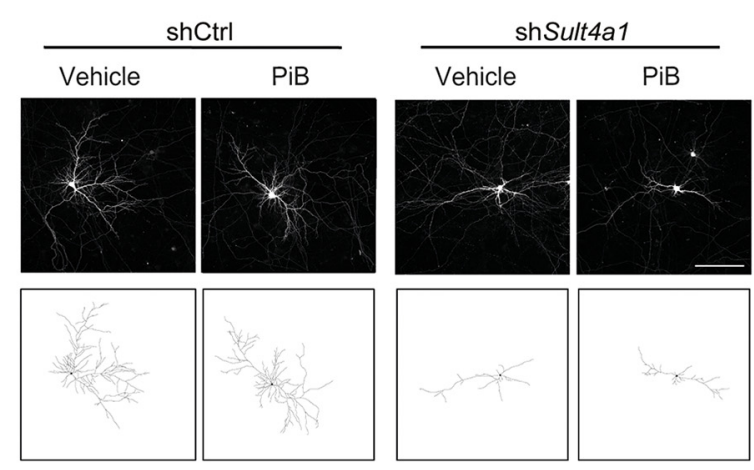

D
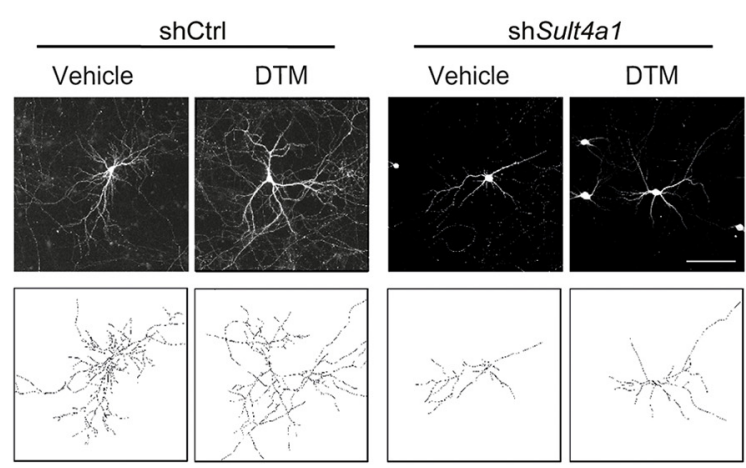

B

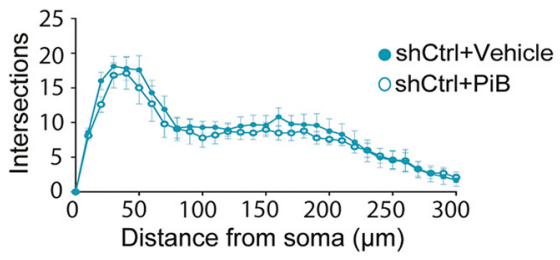

C

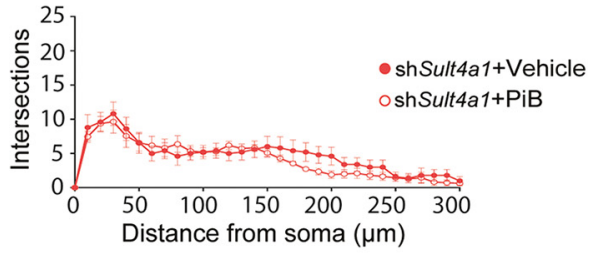

$\mathbf{E}$

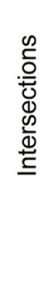
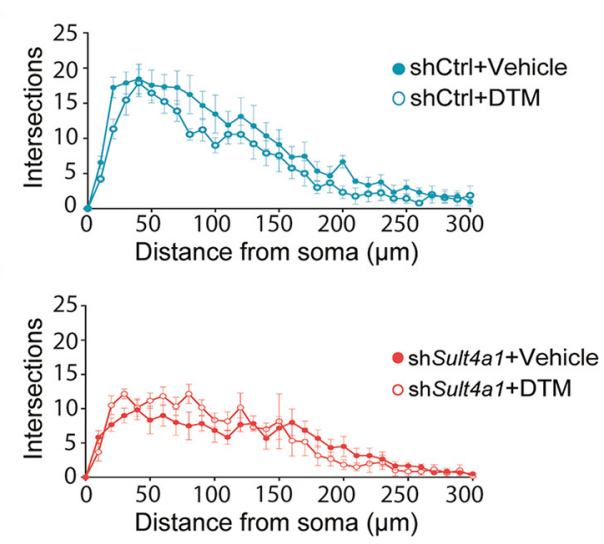

Figure 7. Pin1 pharmacological inhibition does not rescue dendritic arborization after shSult4a1 knockdown. $A$, Representative immunofluorescence images and relative traces of rat cortical neurons transfected with shCtrl or shSult4a1 and treated every other day with $1 \mu \mathrm{m}$ PiB or vehicle, starting at DIV7. Scale bar, $100 \mu \mathrm{m}$. $\boldsymbol{B}, \boldsymbol{C}$, Results of Sholl analysis and quantification of the number of intersections, plotted against the distance from the soma. $\operatorname{shCtrl}(\boldsymbol{B})$ and shSult4a1 $(\boldsymbol{C})$ conditions are displayed separately to clearly illustrate the effect of PiB treatment $(\boldsymbol{B}$ : shCtrl + vehicle, $n=10$ individual neurons; shCtrl + PiB, $n=11$ individual neurons; two-way repeated-measures ANOVA, $p=0.9999$; Sidak's post hoc test: $C$ : shSult4a1 + vehicle, $n=5$ individual neurons; shSult4a1 + PiB, $n=11$ individual neurons; two-way repeated-measures ANOVA, $p=0.9671)$. $\boldsymbol{D}$, Representative immunofluorescence images and relative traces of rat cortical neurons transfected with shCtrl or shSult4a1 and treated every other day with $4 \mu \mathrm{m}$ DTM or vehicle, starting at DIV7. Scale bar, $100 \mu \mathrm{m} . \boldsymbol{E}, \boldsymbol{F}$, Results of Sholl analysis and quantification of the number of intersections, plotted against the distance from the soma. shCtrl $(\boldsymbol{E})$ and shSult4a1 $(\boldsymbol{F})$ conditions are displayed separately to clearly illustrate the effect of DTM treatment $(\boldsymbol{E}$ : shCtrl + vehicle, $n=9$ individual neurons; shCtrl + DTM, $n=9$ individual neurons; two-way repeated-measures ANOVA, $p=0.9021$; Sidak's post hoc test; $F$ : shSult4a $1+$ vehicle, $n=6$ individual neurons; shSult4a1 + DTM, $n=6$ individual neurons; two-way repeated-measures ANOVA, $p=0.0886$; Sidak's post hoc test). Data represent the mean \pm SEM.

These results suggest that SULT4A1, through direct interaction with Pin1 in dendritic spines, plays a novel role in regulating dendritic spine maturation and synaptic transmission.

\section{Discussion}

SULT4A1 is a cytosolic sulfotransferase predominantly expressed in the brain and emerging as a genetic factor in a variety of neurodevelopmental diseases. However, the functional role of SULT4A1 within neuronal development is largely unknown since no substrate or biological functions have been identified yet. SULT4A1 mRNA and protein expression were found to increase during mouse neuron and brain development (Alnouti et al., 2006; Hashiguchi et al., 2018; Idris et al., 2019), supporting a role for SULT4A1 in neuronal maturation. Notably, we found that SULT4A1 is not only expressed in cytosol, and mitochondrial and microsomal fractions (Garcia et al., 2018), but is also highly expressed in synaptosomal preparation, which were obtained from mouse cortical neurons (Fig. $5 G$ ).

Sult4a1-KO mice present with a severe and progressive neurologic phenotype, including tremor, absence seizures, and ataxia, resulting in postnatal death (Garcia et al., 2018). In humans, polymorphisms in the SULTAA1 gene have been associated with susceptibility to schizophrenia (Brennan and Condra, 2005) and several intronic polymorphisms were found in patients with worse cognitive performance (Meltzer et al., 2008). Interestingly, these SULT4A1 polymorphisms are believed to lead to a reduction of mRNA translatability (Brennan and Condra, 2005). PMS is a neurologic disorder characterized by global developmental delay and autistic-like behavior (Phelan and McDermid, 2012) because of deletions of the distal long arm of chromosome 22. Although it is widely recognized that the deletion of SHANK3 gene, encoding a scaffold protein of the postsynaptic density (Naisbitt et al., 1999), is the main cause of the PMS neurologic phenotypes (Durand et al., 2007), the wide clinical heterogeneity among PMS patients suggests that the haploinsufficiency of other genes in the 22q13 region, beside SHANK3, might contribute to cognitive and speech deficits associated with PMS. Approximately 30\% of patients with PMS have a deletion encompassing SULT4A1 (Sarasua et al., 2014), and infants with SULT4A1 deletion displayed a developmental quotient lower than that in patients showing two intact SULT4A1 alleles (Zwanenburg et al., 2016). Moreover, Disciglio et al. (2014) proposed SULT4A1 as a gene related to neurologic symptoms of PMS patients. All of this evidence and the high evolutionary conserved sequence in vertebrate brains (Allali-Hassani et al., 2007) 
strengthen the hypothesis that SULT4A1 plays a pivotal role in CNS development and function.

Garcia et al. (2018) showed that SULT4A1 is expressed in the neurite projections of primary cortical neurons. Our morphologic data show that, in cultured neurons and in vivo, SULT4A1 modulates neuronal branching complexity and dendritic spine density, suggesting a role of SULT4A1 in neuronal maturation and synaptic plasticity. Deficits in dendritic arborization and spine density have been characterized in Shank3 KO mice model of PMS and in mouse models of schizophrenia (Peça et al., 2011; Verpelli et al., 2011; Glausier and Lewis, 2013; Sala and Segal, 2014; Russell et al., 2018; Gouder et al., 2019). Interestingly, we reported alteration in dendritic arborization and spine density both when we silence or overexpress SULT4A1 (Figs. 2, 3). These observations support expression of SULT4A1 at the appropriate level as crucial to ensure proper neuronal development and function. Thus, deficits in SULT4A1 expression, and the resulting decrease in arborization and spine density, may confer developmental deficits that contribute to the presentation or severity of PMS and schizophrenia.

Electrophysiological recordings in primary neurons showed that SULT4A1 modulates NMDAR-mediated synaptic transmission. SULT4A1 silencing induced a decrease in NMDA receptor subunit GluN1 expression (Figs. 4B, 5G) and synaptic localization (Fig. $5 C-G$ ), which were associated with a significant reduction in NMDA current amplitude (Fig. 5A).

The peptidyl-prolyl cis-trans isomerase Pin1, highly expressed in neuronal cells, has been identified as an interactor of SULT4A1 (Smet et al., 2005; Mitchell and Minchin, 2009). However, the physiological role of this interaction is still unknown. Pin 1 is a key regulator of synaptic plasticity (Antonelli et al., 2016), playing a central role in the prolyl isomerization of PSD-95. This function has been shown to negatively affect the formation of the PSD-95/NMDAR complex, which is essential for targeting NMDARs to synapses (Antonelli et al., 2016). This role is supported by the observation that neurons derived from $\mathrm{Pin} 1^{-1-}$ mice exhibit increased spine density and synaptic GluN1 and GluN2B content (Antonelli et al., 2016). In the absence of SULT4A1, we found a higher amount of Pin1 at synaptic sites associated with lower levels of GluN1 and GluN2B (Fig. $5 G$ ), as well as a decreased GluN1/PSD-95 colocalization (Fig. $5 C-F$ ). Considering the known catalytic activity of Pin1 toward PSD-95 (Antonelli et al., 2016), it is compelling to note that in coimmunoprecipitation experiments from SULT4A1 knockeddown cortical neurons we detected an enhanced Pin1/PSD-95 complex formation (Fig. 5H). These results suggest that PSD-95targeted prolyl isomerization mediated by Pinl may be enhanced by SULT4A1 knockdown.

Notably, reduced NMDAR-mediated synaptic transmission caused by the absence of SULT4A1 expression can be rescued through pharmacological inhibition of Pin1 catalytic activity (Fig. $6 A, B$ ). In line with these results, Pin1 inhibition rescues the dendritic spine deficits in SULT4A1 knocked-down neurons (Fig. 6C,D). These results support a role for SULT4A1 in the regulation of PSD-95-targeted Pin1 activity, which in turn controls GluN1 synaptic levels and the formation or stability of dendritic spines. As NMDAR function is intricately linked to synaptic maturation and activity, it is compelling to hypothesize a direct link among GluN1 synaptic levels, dendritic spine density, and the well characterized behavioral deficits within SULT4A1 knock-out models (Crittenden et al., 2015; Garcia et al., 2018).

In conclusion, the present study reveals a novel role for SULT4A1 in modulating neuronal morphology and synaptic activity. We propose a model in which SULT4A1 acts to sequester Pin 1 from the synapse, preventing its isomerase activity toward PSD-95. As PSD-95 prolyl-isomerization has been characterized to negatively modulate the synaptic content of NMDA receptors (Antonelli et al., 2016), this sequestration facilitates the formation of PSD-95/NMDAR complexes within dendritic spines, resulting in increased NMDAR-mediated synaptic activity and dendritic spine maturation (Fig. $6 E$ ).

\section{References}

Allali-Hassani A, Pan PW, Dombrovski L, Najmanovich R, Tempel W, Dong A, Loppnau P, Martin F, Thornton J, Thonton J, Edwards AM, Bochkarev A, Plotnikov AN, Vedadi M, Arrowsmith CH (2007) Structural and chemical profiling of the human cytosolic sulfotransferases. PLoS Biol 5:e97.

Alnouti Y, Petrick JS, Klaassen CD (2006) Tissue distribution and ontogeny of organic cation transporters in mice. Drug Metab Dispos 34:477-482.

Antonelli R, De Filippo R, Middei S, Stancheva S, Pastore B, AmmassariTeule M, Barberis A, Cherubini E, Zacchi P (2016) Pin1 modulates the synaptic content of NMDA receptors via prolyl-isomerization of PSD-95. J Neurosci 36:5437-5447.

Blanchard RL, Freimuth RR, Buck J, Weinshilboum RM, Coughtrie MW (2004) A proposed nomenclature system for the cytosolic sulfotransferase (SULT) superfamily. Pharmacogenetics 14:199-211.

Borroni B, Stanic J, Verpelli C, Mellone M, Bonomi E, Alberici A, Bernasconi P, Culotta L, Zianni E, Archetti S, Manes M, Gazzina S, Ghidoni R, Benussi L, Stuani C, Di Luca M, Sala C, Buratti E, Padovani A, Gardoni F (2017) Anti-AMPA GluA3 antibodies in frontotemporal dementia: a new molecular target. Sci Rep 7:6723.

Brennan MD, Condra J (2005) Transmission disequilibrium suggests a role for the sulfotransferase-4A1 gene in schizophrenia. Am J Med Genet B Genet 139B:69-72.

Chen Y, Stevens B, Chang J, Milbrandt J, Barres BA, Hell JW (2008) NS21: re-defined and modified supplement B27 for neuronal cultures. J Neurosci Methods 171:239-247.

Crittenden F, Thomas HR, Parant JM, Falany CN (2015) Activity suppression behavior phenotype in SULT4A1 frameshift mutant zebrafish. Drug Metab Dispos 43:1037-1044.

Disciglio V, Lo Rizzo C, Mencarelli MA, Mucciolo M, Marozza A, Di Marco C, Massarelli A, Canocchi V, Baldassarri M, Ndoni E, Frullanti E, Amabile S, Anderlid BM, Metcalfe K, Le Caignec C, David A, Fryer A, Boute O, Joris A, Greco D, et al. (2014) Interstitial 22q13 deletions not involving SHANK3 gene: a new contiguous gene syndrome. Am J Med Genet A 164A:1666-1676.

Durand CM, Betancur C, Boeckers TM, Bockmann J, Chaste P, Fauchereau F, Nygren G, Rastam M, Gillberg IC, Anckarsäter H, Sponheim E, Goubran-Botros H, Delorme R, Chabane N, Mouren-Simeoni M-C, de Mas P, Bieth E, Rogé B, Héron D, Burglen L, et al. (2007) Mutations in the gene encoding the synaptic scaffolding protein SHANK3 are associated with autism spectrum disorders. Nat Genet 39:25-27.

Falany CN, Xie X, Wang J, Ferrer J, Falany JL (2000) Molecular cloning and expression of novel sulphotransferase-like cDNAs from human and rat brain. Biochem J 346:857-864.

Garcia PL, Hossain MI, Andrabi SA, Falany CN (2018) Generation and characterization of SULT4A1 mutant mouse models. Drug Metab Dispos 46:41-45.

Glausier JR, Lewis DA (2013) Dendritic spine pathology in schizophrenia. Neuroscience 251:90-107.

Gouder L, Vitrac A, Goubran-Botros H, Danckaert A, Tinevez J-Y, AndréLeroux G, Atanasova E, Lemière N, Biton A, Leblond CS, Poulet A, Boland A, Deleuze J-F, Benchoua A, Delorme R, Bourgeron T, CloëzTayarani I (2019) Altered spinogenesis in iPSC-derived cortical neurons from patients with autism carrying de novo SHANK3 mutations. Sci Rep 9:94.

Hashiguchi T, Shindo S, Chen SH, Hong JS, Negishi M (2018) Sulfotransferase $4 \mathrm{~A} 1$ increases its expression in mouse neurons as they mature. Drug Metab Dispos 46:860-864.

Idris M, Butcher NJ, Minchin RF (2019) The MBNL/CELF splicing factors regulate cytosolic sulfotransferase $4 \mathrm{~A} 1$ protein expression during cell differentiation. Drug Metab Dispos 47:314-319. 
Liyou NE, Buller KM, Tresillian MJ, Elvin CM, Scott HL, Dodd PR, Tannenberg AE, McManus ME (2003) Localization of a brain sulfotransferase, SULT4A1, in the human and rat brain: an immunohistochemical study. J Histochem Cytochem 51:1655-1664.

Lois C, Hong EJ, Pease S, Brown EJ, Baltimore D (2002) Germline transmission and tissue-specific expression of transgenes delivered by lentiviral vectors. Science 295:868-872.

Meltzer HY, Brennan MD, Woodward ND, Jayathilake K (2008) Association of Sult4A1 SNPs with psychopathology and cognition in patients with schizophrenia or schizoaffective disorder. Schizophr Res 106:258-264.

Minchin RF, Lewis A, Mitchell D, Kadlubar FF, McManus ME (2008) Sulfotransferase 4A1. Int J Biochem Cell Biol 40:2686-2691.

Mitchell DJ, Minchin RF (2009) Cytosolic Aryl sulfotransferase 4A1 interacts with the peptidyl prolyl cis-trans isomerase Pin1. Mol Pharmacol 76:388395.

Mitz AR, Philyaw TJ, Boccuto L, Shcheglovitov A, Sarasua SM, Kaufmann WE, Thurm A (2018) Identification of 22q13 genes most likely to contribute to Phelan McDermid syndrome. Eur J Hum Genet 26:293-302.

Naisbitt S, Kim E, Tu JC, Xiao B, Sala C, Valtschanoff J, Weinberg RJ, Worley PF, Sheng M (1999) Shank, a novel family of postsynaptic density proteins that binds to the NMDA receptor/PSD-95/GKAP complex and cortactin. Neuron 23:569-582.

Naldini L, Blömer U, Gallay P, Ory D, Mulligan R, Gage FH, Verma IM, Trono D (1996) In vivo gene delivery and stable transduction of nondividing cells by a lentiviral vector. Science 272:263-267.

Negishi M, Pedersen LG, Petrotchenko E, Shevtsov S, Gorokhov A, Kakuta Y, Pedersen LC (2001) Structure and function of sulfotransferases. Arch Biochem Biophys 390:149-157.

Osborn M, Weber K (1982) Immunofluorescence and immunocytochemical procedures with affinity purified antibodies: tubulin-containing structures. Methods Cell Biol 24:97-132.

Peça J, Feliciano C, Ting JT, Wang W, Wells MF, Venkatraman TN, Lascola CD, Fu Z, Feng G (2011) Shank3 mutant mice display autistic-like behaviours and striatal dysfunction. Nature 472:437-442.

Phelan K, McDermid HE (2012) The 22q13.3 deletion syndrome (PhelanMcDermid syndrome). Mol Syndromol 2:186-201.

Ramsey TL, Meltzer HY, Brock GN, Mehrotra B, Jayathilake K, Bobo WV, Brennan MD (2011) Evidence for a SULT4A1 haplotype correlating with baseline psychopathology and atypical antipsychotic response. Pharmacogenomics 12:471-480.

Ranganathan R, Lu KP, Hunter T, Noel JP (1997) Structural and functional analysis of the mitotic rotamase Pin 1 suggests substrate recognition is phosphorylation dependent. Cell 89:875-886.

Russell TA, Grubisha MJ, Remmers CL, Kang SK, Forrest MP, Smith KR, Kopeikina KJ, Gao R, Sweet RA, Penzes P (2018) A schizophrenia-linked KALRN coding variant alters neuron morphology, protein function, and transcript stability. Biol Psychiatry 83:499-508.

Sala C, Segal M (2014) Dendritic spines: the locus of structural and functional plasticity. Physiol Rev 94:141-188.
Sarasua SM, Dwivedi A, Boccuto L, Chen CF, Sharp JL, Rollins JD, Collins JS, Rogers RC, Phelan K, DuPont BR (2014) 22q13.2q13.32 genomic regions associated with severity of speech delay, developmental delay, and physical features in Phelan-McDermid syndrome. Genet Med 16:318-328.

Sessa A, Mao CA, Hadjantonakis AK, Klein WH, Broccoli V (2008) Tbr2 directs conversion of radial glia into basal precursors and guides neuronal amplification by indirect neurogenesis in the developing neocortex. Neuron 60:56-69.

Shen M, Stukenberg PT, Kirschner MW, Lu KP (1998) The essential mitotic peptidyl-prolyl isomerase Pin 1 binds and regulates mitosis-specific phosphoproteins. Genes Dev 12:706-720.

Sidharthan NP, Butcher NJ, Mitchell DJ, Minchin RF (2014) Expression of the orphan cytosolic sulfotransferase SULT4A1 and its major splice variant in human tissues and cells: dimerization, degradation and polyubiquitination. PLoS One 9:e101520.

Smet C, Wieruszeski JM, Buée L, Landrieu I, Lippens G (2005) Regulation of Pin 1 peptidyl-prolyl cis/trans isomerase activity by its WW binding module on a multi-phosphorylated peptide of Tau protein. FEBS Lett 579:4159-4164.

Tatara Y, Terakawa T, Uchida T (2010) Identification of Pin1-binding phosphorylated proteins in the mouse brain. Biosci Biotechnol Biochem 74:2480-2483.

Verpelli C, Piccoli G, Zibetti C, Zanchi A, Gardoni F, Huang K, Brambilla D, Di Luca M, Battaglioli E, Sala C (2010) Synaptic activity controls dendritic spine morphology by modulating eEF2-dependent BDNF synthesis. J Neurosci 30:5830-5842.

Verpelli C, Dvoretskova E, Vicidomini C, Rossi F, Chiappalone M, Schoen M, Di Stefano B, Mantegazza R, Broccoli V, Böckers TM, Dityatev A, Sala C (2011) Importance of Shank3 protein in regulating metabotropic glutamate receptor 5 (mGluR5) expression and signaling at synapses. J Biol Chem 286:34839-34850.

Vicidomini C, Ponzoni L, Lim D, Schmeisser MJ, Reim D, Morello N, Orellana D, Tozzi A, Durante V, Scalmani P, Mantegazza M, Genazzani AA, Giustetto M, Sala M, Calabresi P, Boeckers TM, Sala C, Verpelli C (2017) Pharmacological enhancement of mGlu5 receptors rescues behavioral deficits in SHANK3 knock-out mice. Mol Psychiatry 22:689-702.

Wiznerowicz M, Trono D (2003) Conditional suppression of cellular genes: lentivirus vector-mediated drug-inducible RNA interference. J Virol 77:8957-8961.

Ziats CA, Grosvenor LP, Sarasua SM, Thurm AE, Swedo SE, Mahfouz A, Rennert OM, Ziats MN (2019) Functional genomics analysis of PhelanMcDermid syndrome 22q13 region during human neurodevelopment. PLoS One 14:e0213921.

Zwanenburg RJ, Bocca G, Ruiter SA, Dillingh JH, Flapper BC, van den Heuvel ER, van Ravenswaaij-Arts CM (2016) Is there an effect of intranasal insulin on development and behaviour in Phelan-McDermid syndrome? A randomized, double-blind, placebo-controlled trial. Eur J Hum Genet 24:1696-1701. 